Portland State University

PDXScholar

Spring 7-11-2018

\title{
Short-Term Tracking of Orientation with Inertial Sensors
}

Golriz Sedaghat

Portland State University

Follow this and additional works at: https://pdxscholar.library.pdx.edu/open_access_etds

Part of the Biomedical Commons

Let us know how access to this document benefits you.

\section{Recommended Citation}

Sedaghat, Golriz, "Short-Term Tracking of Orientation with Inertial Sensors" (2018). Dissertations and Theses. Paper 4467.

https://doi.org/10.15760/etd.6351

This Thesis is brought to you for free and open access. It has been accepted for inclusion in Dissertations and Theses by an authorized administrator of PDXScholar. Please contact us if we can make this document more accessible: pdxscholar@pdx.edu. 
Short-Term Tracking of Orientation with Inertial Sensors

by
Golriz Sedaghat

A thesis submitted in partial fulfillment of the requirements for the degree of

\author{
Master of Science \\ in \\ Electrical and Computer Engineering
}

Thesis Committee

James McNames, Chair

Martin Siderius

Peter Jacobs

Portland State University

2018 


\section{Abstract}

In the past several years, IMU's have been widely used to measure the orientation of a moving body over a continuous period of time. Although, inertial navigation is a common approach for estimating the orientation, it greatly suffers from the accumulation of error in the orientation estimation. Most of the current common practices apply zero velocity update as a calibration method to address this problem and improve the estimation accuracy. However, this approach requires the sensors to be stationary frequently.

This thesis introduces a novel method of calibration for estimating the elevation and bank angles of the orientation over a persistent human movement utilizing accelerometers and gyroscopes. The proposed technique incorporates the prior knowledge about the human motion to the estimation of the orientation to prevent the estimated position from growing unboundedly. The measurement model is designed to estimate the position for $T$ seconds in the future. The knowledge of the estimated position for few seconds further in the future provides a feedback for orientation estimation during the periods of time when the accelerometer's readings are significantly deviated from gravity.

This work evaluates the performance of the proposed method in two different ways: 1. a model of human movement is designed to generate synthetic data which resembles human motion. 2. an experimental design is implemented using a robot arm and an actual IMU to capture real data. The performance of 
the new technique is compared with the results from the inertial navigation approach. It is demonstrated that the new method significantly improves the accuracy of the orientation estimation. 


\section{Dedication}

This thesis is dedicated to thousands of students in Iran, including my two sisters, who are denied from formal university education due to their religious or ideological beliefs. 


\section{Acknowledgements}

I would like to thank my advisor, Dr. James McNames, for all your support, encouragement and generosity in sharing your knowledge. I appreciate you teaching me how to research and develop a scientific thought. Your high standards, although at first might have appeared difficult, has been and will continue to be the way for growth and progress in my life.

I also would like to express my gratitude to the Biomedical Signal Processing lab at Portland State University for making some of the latest technology accessible to the students. In particular, thanks to Paul Vasilyev for his kind help with working with the robot arm and collecting data.

Finally, I owe my sincere appreciation to my family for their unfailing support and continuous encouragement throughout my life. My mom for her unconditional love and sacrifice, my dad for his patient and gentle example, my sisters, Shidrokh and Raha, although far away from me, they are always in my heart and every minute of my life. This accomplishment would not have been possible without them. 


\section{Contents}

Abstract $\quad$ i

Dedication $\quad$ iii

Acknowledgements $\quad$ iv

List of Tables viii

List of Figures $\quad$ ix

1 Introduction $\quad 1$

1.1 Motion capture . . . . . . . . . . . . . . . . . 1

1.1.1 Virtual Reality . . . . . . . . . . . . . . 1

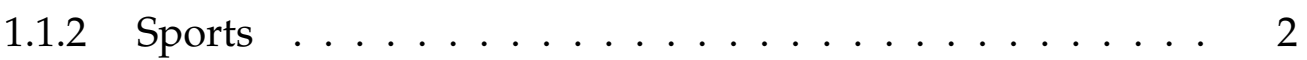

1.1.3 Clinical Research and Practice ............ 2

1.1.4 Entertainment ................. 3

1.2 Motion Capture Technologies . . . . . . . . . . . . 4

1.2.1 Optical Motion Capture . . . . . . . . . . . . . . . . 4

1.2.2 Mechanical Motion Capture ............ 5

1.2.3 Magnetic Motion Capture ............. 5

1.2.4 Acoustic Motion Capture ............. 6

1.2.5 Inertial Motion Capture . . . . . . . . . . . 6 


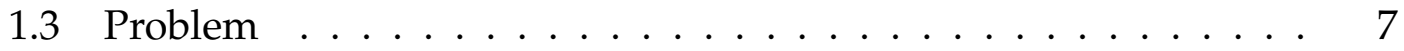

1.4 Objective and contributions $\ldots \ldots \ldots \ldots \ldots$

1.5 Thesis Overview $\ldots \ldots \ldots \ldots$

2 Literature Review 10

2.1 Limited by the Duration of Tracking and Controlled Movement . 10

2.2 Limited by the Controlled Environment . . . . . . . . . . . . . 13

2.3 Limited by the Translational Acceleration . . . . . . . . . . . 14

2.4 Limitations of Previous Studies . . . . . . . . . . . . . 18

3 Tracker Design $\quad 19$

3.1 Bayesian Framework . . . . . . . . . . . . . . . . . . . . . 19

3.2 Nonlinear State Space Model _. . . . . . . . . . . . . . . . . 21

3.3 Estimation and Filtering $\ldots \ldots \ldots \ldots \ldots$

3.3.1 Kalman Filter and Its Variations $\ldots \ldots \ldots$

3.4 Process and Measurement Models . . . . . . . . . . . . . . . 24

3.4.1 Quaternions . . . . . . . . . . . . . . . 24

3.4.2 Quaternion Rotation Operator . . . . . . . . . . . 25

3.4 .3 Euler Angles . . . . . . . . . . . . . . . . . . 26

3.4 .4 Process Model . . . . . . . . . . . . . . . . . . . . . 27

3.4.5 Measurement Model . . . . . . . . . . . . . . . . . . 28

3.5 Filter Design . . . . . . . . . . . . . . . . . . . . 30

3.5.1 UKF Design Parameters . . . . . . . . . . . . . . . 32

4 Performance Assessment $\quad 35$

4.1 Model Validation Using Synthetic Data $\ldots \ldots$. . . . . . . . 35 
4.1.1 Design of Synthetic Data . . . . . . . . . . . . . 36

Design Goals for Modeling Human Motion . . . . . . . . . 36

Design Decisions for Modeling Human Motion . . . . . 38

4.2 Experimental Design . . . . . . . . . . . . . . . . . 45

4.2.1 Robot Arm . . . . . . . . . . . . . . . . . 45

4.2.2 Data Preprocessing . . . . . . . . . . . . . . . . . . . . 49

Frames of Reference Alignment . . . . . . . . . . . . . 50

Removal of Abrupt Changes from the Robot Arm Euler Angles . . . . . . . . . . . . . . 50

Resampling the Robot Arm Measurements . . . . . . . . 52

Removal of Delay Between the Robot and Opal Measure-

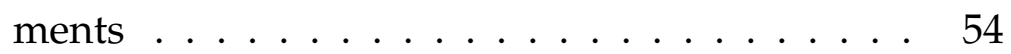

4.3 Euler Angles Performance Assessment . . . . . . . . . . . . . 55

5 Results and Discussion $\quad 57$

5.1 Algorithm Performance Using Synthetic Data . . . . . . . . . . 57

5.2 Algorithm Performance Using Real Data . . . . . . . . . . 60

6 Conclusion $\quad 65$

6.1 Summary . . . . . . . . . . . . . . . . 65

6.2 Contribution .................... 66

6.3 Limitations and Future Research . . . . . . . . . . . . . 67

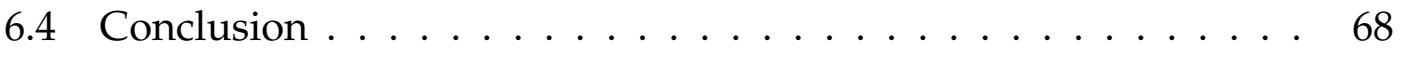

$\begin{array}{ll}\text { Bibliography } & 70\end{array}$ 


\section{List of Tables}

1.1 Advantages and disadvantages of motion capture technologies . 8

3.1 User-specified parameters of UKF design . . . . . . . . . . . 34

4.1 Epson Robots, Carson, California . . . . . . . . . . . . . 47

5.1 Median absolute deviation of the elevation and bank angles for synthetic data . . . . . . . . . . . . . . . . . . 59

5.2 Median absolute deviation of the elevation and bank angles for the real data . . . . . . . . . . . . . . . . . . . 64 


\section{List of Figures}

4.1 Block diagram of design of human movement properties . . . . . 38

4.2 Velocity standard deviation vs the location of the high-pass filter pole ........................... 40

4.3 Position standard deviation vs the location of the high-pass filter pole ........................... 40

4.4 Top, middle and bottom panels demonstrate synthetic acceleration, velocity and position respectively. . . . . . . . . . . . . 41

4.5 Block diagram of the design of the angular velocity, $\omega_{n} \ldots \ldots 2$

4.6 Angular velocity standard deviation vs $\alpha \ldots \ldots$. . . . . . . . 43

4.7 Angular velocity standard deviation vs White noise power . . . . 44

4.8 Top: Synthetic Angular Velocity, Bottom: Synthetic Bank Angle 45

4.9 Epson C3 Robot Arm . . . . . . . . . . . . . . . 46

4.10 Opal Sensor . . . . . . . . . . . . . . . . . . . 47

4.11 Robot End effector . . . . . . . . . . . . . . . . . 49

4.12 Robot Arm Orientation . . . . . . . . . . . . . . . . . . . 49

4.13 Robot Arm Coordinate System . . . . . . . . . . . . . . 51

4.14 Opal Sensor Coordinate System . . . . . . . . . . . . . 51

4.15 Overlap of the original robot Euler angles (light, thick blue) and resampled Euler angles (dark, thin blue) . . . . . . . . . . . 53 
4.16 Cross-correlation of Opal and robot Euler angles . . . . . . . . 55

5.1 Median absolute error between the true and estimated Euler angles: the blue line represents the MAD between the true and estimated angles by inertial navigation method for 50 realizations, the red line shows the MAD between the true and estimated angles measured by the new technique for 10 realizations. . . . . . . 58

5.2 Comparison of performance of inertial navigation approach with the short-term tracking algorithm for different number of seconds in the future on the synthetic data. . . . . . . . . . . . . 59

5.3 True elevation (top panel) and bank (bottom panel) angles . . . . 60

5.4 Estimated elevation (top panel) and bank (bottom panel) angles by short-term tracking algorithm . . . . . . . . . . . 61

5.5 Estimated elevation (top panel) and bank (bottom panel) angles by inertial navigation approach . . . . . . . . . . . . . 61

5.6 The overlay of the true and estimated elevation (top panel) and bank (bottom panel) angles. The black lines show the true angles and the magnum lines illustrate the estimated angles using the short-term tracking algorithm. . . . . . . . . . . . 62

5.7 The overlay of the true and estimated elevation (top panel) and bank (bottom panel) angles. The black lines show the true angles and the red lines illustrate the estimated angles using the inertial

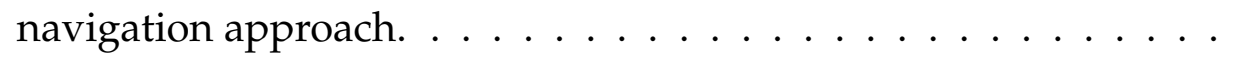


5.8 Comparison of performance of inertial navigation approach with the short-term tracking algorithm for different number of seconds in the future on the real data. . . . . . . . . . . . . 64 


\section{Introduction}

This chapter provides an introduction to the topic of the motion capture. Section 1.1 introduces several common applications of motion capture technologies in various fields. Section 1.2 describes the variety of technologies used in tracking and capturing the motion and lists some of the strengths and limitations of each approach. Ultimately, Section 1.3 provides an overview of the problem which this thesis attempts to address.

\subsection{Motion capture}

Motion capture is the process of capturing and recording the motion of a moving body. With the advancement of technology, motion capture has applications in various fields of research including virtual reality, sports, clinical practice and clinical research as well as entertainment.

\subsubsection{Virtual Reality}

Virtual reality technology provides the user the psychological experience of being in a virtual environment which is generated by a computer. These technologies use headsets to simulate a virtual environment by creating realistic images, sounds, vibrations and other sensations. Virtual reality has applications in military and commercial aviation and medical trainings (Dam et al., 2000). 
Advancements in the motion capture technologies have made it feasible to accurately track movements and render the movements in a virtual world. Virtual reality technologies are utilized as a tool to learn and develop skills before executing the action in the real world. For example, computer-based simulators are used to improve patient safety in crisis management scenarios as well as to train surgeons the essential technical skills (Salas et al., 2005). Virtual reality systems are generally combined with inertial sensors to avoid occlusion problems and increase measurement accuracy.

\subsubsection{Sports}

The other application of motion capture is in the professional sports to detect the movement of athletes. Analysis of changes in joint angles can provide useful information to help fine-tuning the athletic techniques and improve their performance (El-Gohary, 2013). Inertial measurement units have been used to directly measure the changes of the joint angles by placing them on various segments of an athlete's body. The data gathered by the motion capture technologies helps the researchers to further their understanding of the mechanism of the motion to prevent injuries and help with rehabilitation from the injuries (Lapinski, 2008).

\subsubsection{Clinical Research and Practice}

The application of real-time motion capture in health care including clinical work and therapy is significantly increasing. Motion tracking helps with understanding the human motor behavior, diagnosis of neurological movement 
disorders and development of treatments and techniques to improve the performance of the human movement.

Clinicians often assess the therapies by taking the history and by performing clinical examinations which only capture a short activity of the patient while the symptoms usually differ day by day. Moreover, the clinical observations are subjective. Motion capture techniques provide objective long-term, ambulatory approaches to monitor the patient symptoms. Furthermore, movement analysis helps to restore the impaired mobility of patients suffering from stroke or brain injuries. Physical therapists use motion capture technologies to evaluate gait and general body movement and ultimately choose the right treatment based on the individual condition. In addition, motion capture technologies are utilized to optimize the therapies by quantifying the effectiveness of the treatment.

\subsubsection{Entertainment}

In the past decades, digital entertainment has utilized motion capture technologies to track the motion of the human for creation of visual effects for movies and video games as well as generation of animation of animals and creatures (Lun et al., 2010). Furthermore, the application of virtual reality for entertainment purposes requires implementation of motion capture systems which reflect the motions of the player in the virtual world by tracking and analyzing the movements of the player (Zhang et al., 2011). 


\subsection{Motion Capture Technologies}

A variety of technologies such as optical, mechanical, magnetic, acoustic, and inertial sensors are available to measure the human movement. Tracking algorithms are used to analyze and process the collected measured data by different sensors. The processing is done either in real time or offline based on the application.

\subsubsection{Optical Motion Capture}

Optical motion capture systems use a number of cameras to observe the moving object from different angles to allow reconstructing the subject in three dimensions. There are three different methods of capturing motion using optical technologies, "active marker-based or pulsed-LED", "passive marker-based or reflective" and "marker-less".

In marker-based approach, motion is captured by a number of cameras viewing a set of markers attached to the moving body. Optical filters adjusted to the wavelength of the radiant source are placed on the cameras. The active method uses LED markers which pulse synchronously with the camera's shutters while the passive systems apply retro-reflective markers which reflect the light emitted by the radiant sources located next to the cameras. Active markers are identifiable by modulating their pulses while passive markers are presumed by continuous observation (Kirk, O'Brien, and Forsyth, 2005).

In marker-less method, the three dimensional body is reconstructed using an outline of the moving object obtained by the cameras which view the subject 
from different angles.

Although optical systems are very accurate for tracking the movement, their application is limited to the laboratory environments where there is a clear line of sight between the source and the sensor.

\subsubsection{Mechanical Motion Capture}

In mechanical motion capture, a skeletal-like structure is worn to the moving body to directly measure the body joint angles. The structure consists of the jointed mechanical parts connected together with electromechanical transducers whose shapes change as the body moves. While the mechanical motion capture techniques can provide precise and free of occlusion estimates of the movement of a joint, their range of motion is limited and they are inconvenient to be used for monitoring daily activities (El-Gohary, 2013).

\subsubsection{Magnetic Motion Capture}

Magnetic motion capture systems measure the strength of the magnetic fields generated by three orthogonal magnetic sensors in a transmitter source. A processor calculates position and orientation of each sensor. Although magnetic systems do not suffer from occlusion, they can be disturbed by magnetic materials in the environment ( $\mathrm{O}^{\prime}$ Brien et al., 2000). 


\subsubsection{Acoustic Motion Capture}

In acoustic motion capture systems, ultrasonic pulses, sent by the emitters, are used to determine the position using methods such as phase coherence, time-offlight or triangulation. The acoustic systems are limited by the physics of sound such as sensitivity to temperature and humidity changes as well as sound reflections by the environment. Necessity of having a clear line of sight between the emitter and the transmitter is another major limitation of the acoustic systems (Hermann, Höner, and Ritter, 2006).

\subsubsection{Inertial Motion Capture}

Inertial motion capture systems utilize Inertial Measurement Units (IMU) to measure motion. IMU's have broad applications in various fields such as human interfaces for multimedia appliances based on body gesture, electronic stability control technologies, inertial navigation systems (INS) and medical field applications related to movement analysis. IMU's are widely used due to their rapid advancements in terms of technology, functionality, cost, size and real time applications. Wearable IMU's can be attached to different body segments to capture and record the movement. The IMU's consist of accelerometers and gyroscopes. The angular velocity measured by gyroscopes are integrated over time to compute the change in angle with respect to a known initial angle. Accelerometer measures gravitational and translational acceleration. Gravity vector is obtained by transformation of the acceleration from the sensor frame to earth frame. The acceleration is integrated once to compute the velocity and then again to compute the position of the object. This method is well known 
as inertial navigation; the major problem with this approach is the cumulative measurement errors over time which results in a growing drift in estimation of the orientation and position. Tracking algorithms such as Kalman filters are used to process the collected data (Barbour and Schmidt, 2001). Table 1.1 summarizes the advantages and disadvantages of various motion capture technologies introduced in this chapter.

\subsection{Problem}

The application of the IMU's to measure the orientation of a moving body continuously and over an extended period of time has been increasing due to the low cost and realtime applications of the inertial measurement units. A typical approach to estimate the orientation of a body using IMU's is to integrate the angular rate measured by the gyroscopes and double integrate the acceleration measured by the accelerometers to compute the orientation and the position of the object respectively. The main issue with this approach is the accumulation of error when integrating the sensor measurements which results in inaccurate estimation of the orientation and the position. Previous studies have shown that integrating the noisy measurements from the gyroscopes can create the orientation errors of up to 10-25 degrees after one minute (Roetenberg, 2006) and the position estimation errors obtained from integrating the acceleration measured by the accelerometers would grow with the cube of time (Foxlin, 2005).

\subsection{Objective and contributions}

This thesis designs, develops and rigorously assesses a more accurate algorithm to estimate the orientation of the human movement over a persistent motion using accelerometers and gyroscopes. 


\begin{tabular}{|c|c|c|}
\hline \multicolumn{3}{|c|}{ Advantages and disadvantages of motion capture technologies } \\
\hline Technologies & Advantages & Disadvantages \\
\hline Optical & $\begin{array}{l}\text { High sampling rate, } \\
\text { high accuracy, } \\
\text { Unlimited movement range }\end{array}$ & $\begin{array}{l}\text { Occlusion, } \\
\text { provides only positional } \\
\text { data, } \\
\text { expensive }\end{array}$ \\
\hline Mechanical & $\begin{array}{l}\text { Accurate estimates of single } \\
\text { joint angles, } \\
\text { inexpensive, } \\
\text { not dependent on external } \\
\text { factors, } \\
\text { doesn't suffer from occlusion }\end{array}$ & $\begin{array}{l}\text { Limited by mechanical con- } \\
\text { straints, } \\
\text { limited motion range, } \\
\text { hard to monitor daily activi- } \\
\text { ties, } \\
\text { equipment needs recalibra- } \\
\text { tion }\end{array}$ \\
\hline Magnetic & $\begin{array}{l}\text { Real-time tracking, } \\
\text { low computational cost, } \\
\text { low cost }\end{array}$ & $\begin{array}{l}\text { Limited range of motion, } \\
\text { Sensitive to any magnetic } \\
\text { field }\end{array}$ \\
\hline Acoustic & $\begin{array}{l}\text { Transmitter can be placed on } \\
\text { a body segment or fixed in } \\
\text { the measurement volume, } \\
\text { lack of occlusion in the case of } \\
\text { multiple users }\end{array}$ & $\begin{array}{l}\text { Physics of sound limits the } \\
\text { accuracy, sampling rate and } \\
\text { the range of acoustic } \\
\text { sensitive to sound reflection }\end{array}$ \\
\hline Inertial & $\begin{array}{l}\text { Inexpensive, } \\
\text { can be used in and outside of } \\
\text { laboratory, } \\
\text { suitable for monitoring daily } \\
\text { activities, } \\
\text { not dependent on external } \\
\text { factors, } \\
\text { unlimited range of motion }\end{array}$ & $\begin{array}{l}\text { Positional drift, } \\
\text { accumulation of measure- } \\
\text { ment error over time }\end{array}$ \\
\hline
\end{tabular}

TABLE 1.1: Advantages and disadvantages of motion capture technologies 


\subsection{Thesis Overview}

The thesis is organized as follows:

Chapter 2 reviews the previous work upon which the research draws.

Chapter 3 describes the design methodology.

Chapter 4 evaluates the performance of the algorithm using synthetic and real data.

Chapter 5 discusses the results of the performance of the algorithm using the synthetic and real data.

Chapter 6 reviews the contributions of the thesis, and lists the possible directions of future research. 


\section{Literature Review}

Numerous studies have been conducted to address the accumulation of the measurement error in the estimation of the orientation and position of human movement obtained from integrating the gyroscopes and accelerometers measurements respectively over time also known as the drift problem. The rest of this section introduces some of the common and latest studies on this matter. The literature review was performed based on searching for keywords of inertial sensors and human motion tracking and/or magnetometers in digital libraries including IEEE Xplore and Web of Science.

\subsection{Limited by the Duration of Tracking and Controlled Movement}

This section summarizes some of the papers in which the tracking algorithm is limited by the type of the movement and by its ability to prevent the growth of the drift for only a short period of time.

Rehbinder et al. used accelerometers and gyroscopes to estimate the orientation of a walking robot relative to the gravity vector particularly during the large and abrupt changes in the acceleration. They used the rotation matrices to represent the rotations of the rigid body to be able to use linear formulations. In order to address the problem with the inaccurate accelerometer measurements during the fast movements, they designed a switching architecture with two modes, one for low and one for high accelerations where they tuned the noise parameters based on the estimated acceleration level. The approach was only examined in a simulating environment and the results were reported for a 12-second long motion consisting of 4 seconds of high accelerations and 1 second of 
zero acceleration patterns. The results showed that although the orientation estimation error increased during the high acceleration phases, during the low acceleration phases the error was compensated for (Rehbinder and $\mathrm{Hu}, 2001$ ). However, the assessment of the algorithm was done in a simulating environment with a controlled pattern of motion for a short period of time.

Roetenberg et al. fused the measurements from three gyroscopes, three accelerometers and three magnetometers into a complementary Kalman filter to estimate the orientation of human body segments. The filter estimated the gyroscope bias error as well as the orientation and the magnetic disturbances errors. They assumed the gyroscopes measured the angular velocity plus an offset with a lower bandwidth compared to the bandwidth of the kinematic signals, and the accelerometers measured the translational acceleration described as low-pass filtered white noise as well as the gravitational acceleration. The magnetometer measured the earth magnetic field vector plus the disturbances whose bandwidth was larger than the bandwidth of the gyroscope drift. White noise was considered as another additive component of the measured data by all the three types of sensors. The Kalman filter operated based on two inputs; one the difference between the estimates of the inclination by accelerometers and gyroscopes and the second one the difference between the estimates of the global magnetic field vector by gyroscopes and magnetometers. The algorithm was evaluated under quasi-static and dynamic conditions with ferromagnetic materials close to the sensor. The quasi-static case was defined as having static positions and rotations about the three axes. Although the results showed using the magnetic interference corrections significantly improved the orientation estimates, the algorithm was tested under controlled and limited conditions, and the accuracy of the estimations depended on the speed and type of the movement (Roetenberg et al., 2005). 
Giansanti et al. designed a system without gyroscopes and with multiple accelerometers to determine the orientation and position of limbs (Giansanti et al., 2003). They measured the orientation estimation error as 10 degrees for a short one-second interval. In the next study, they added gyroscopes to the system to track the position and orientation over a protocol of stand-to-sit, sit-to-stand and gait-initiation. The estimation error was measured 0.2 degrees over 1.2 seconds time interval for the sit-to-stand movement. Although adding gyroscopes to the system significantly decreased the orientation estimation error, the application of the algorithm was restricted to simple tasks and limited to a short duration of 4 seconds (Giansanti, Maccioni, and Macellari, 2005).

In 2006, and in a subsequent study, Zhou et al. introduced a Kalman-filter-based design consisting of accelerometers, gyroscopes and magnetometers to estimate the orientation of the human upper limb movement. They employed two inertial sensors to independently measure the angle and the position. An experimental work was conducted to assess the performance of the tracker. Two tasks were included in the experiment: first, driving the wrist along a designed path; this task allowed to evaluate the accuracy of the proposed detector. Each task lasted for 20 seconds and the subjects tried to keep their shoulder still during the arm movement. Second, performing five daily activities including reach, hand to mouth, drink, flexion-extension, and elevation each for a period of 20 seconds. Although the authors stated that the algorithm was robust and relatively accurate for different motions, the performance of the tracker was reported for a short time period of 20 seconds. In addition, the authors indicated that the cost of the system remained an issue which needed to be addressed in the future work (Zhou, $\mathrm{Hu}$, and Tao, 2006). 


\subsection{Limited by the Controlled Environment}

This section summarizes some of the papers in which the tracking is limited by the magnetic disturbances in the environment.

Bachmann et al. described a quaternion-based complementary filter using a sensor module consisted of accelerometers, gyroscopes and magnetometers to track the human motion. The application of the quaternions enabled the filter to track all orientations without encountering the singularity problem. The orientation of each limb segment relative to an earth-fixed reference frame was individually determined using a sensor module. The drift on estimating the orientation was corrected without requiring having still periods in the movement; however the accuracy of the system was affected by the presence of the ferromagnetic disturbances (Bachmann, 2000). In a subsequent study, Bachmann et al. attempted to quantify the errors in the orientation estimation caused by the local magnetic field disturbances using a similar sensor module as in the previous work. They showed that variations in the direction of the environmental magnetic field caused errors in the estimation of the heading angle. The reported error due to the changes in the local magnetic disturbances was 16 degrees when the sensor was in a two-feet range of the source of interference (Bachmann, Yun, and Peterson, 2004).

In 2007, and in a subsequent study, Roetenberg et al. designed a portable magnetic system consisted of gyroscopes, accelerometers and magnetometers to estimate the position and orientation of human movement using a complementary Kalman filter. In order to obtain long-term stable assessment of relative positions, the design utilized the magnetic system to provide measurement updates at a relatively low rate $(1-2 \mathrm{~Hz})$. The results were reported for the evaluation of the tracker without the magnetic disturbances in the environment and the authors stated the necessity of the investigation of the performance of the system in the presence of the magnetic fields (Roetenberg et al., 2005). 
Sabatini et al. utilized one single IMU including a biaxial accelerometer and one gyroscope placed on the foot to capture the gait events (heel-off, toe-off, heel-strike, foot-flat). The gyroscope measurements were integrated to compute the elevation angle which was used to determine the transition between the gait phases. The acceleration measurements during the swing of the foot were double integrated to calculate the position of the foot. It was assumed that the motion was in the sagittal plane only since the accelerometer doesn't provide information on the rotations about the vertical axis or the heading angle. The algorithm was only tested during treadmill walking and not in a free-living environment (Sabatini et al., 2005).

Gallagher et al. presented a technique to compute the posture estimates using accelerometers, gyroscopes and magnetometers utilizing the rotation matrices to represent the orientations. A complementary filter was proposed to estimate the posture instead of applying the standard Kalman filter to increase the efficiency of the tracker and avoid designing a process model with multiple states which is computationally expensive. The simple time-domain, first-order complementary filter performed low-pass filtering on the accelerometer and magnetometer signals and high-pass filtering on the gyroscope signal. In order to assess the performance of the system, a PHANToM robot was used to perform some activities lasting for 10 seconds. The developed technique computed accurate posture estimates at an update frequency of $125.75 \mathrm{KHz}$; however, the accuracy of the algorithm was sensitive to the presence of the magnetic fields and the system was reported to track the motion for a short duration of 10 seconds only (Gallagher, Matsuoka, and Ang, 2004).

\subsection{Limited by the Translational Acceleration}

This section summarizes some of the papers in which the tracking is limited to movement with small translational accelerations. 
Luinge et al. used the inclination information from the accelerometers to improve the orientation estimates obtained from integrating the rotational rate from the gyroscopes (Luinge, Veltink, and Baten, 1999; Luinge, 2002). They suggested to split the obtained orientation into tilt (also known as elevation and bank angles) and orientation about the vertical axis (also known as heading angle). The tilt during the static periods was calculated from the gravitational acceleration. To improve the accuracy of the tilt angle estimation, the difference between gyroscope and accelerometer tilt was fused into a Kalman filter. The tilt estimates and the measured angles of rotation about the vertical axis were combined to produce more accurate orientation angles. The main limitation of this method was that the estimates were accurate only for brief periods when the subject was not moving and the acceleration measurements were only due to gravity (Luinge and Veltink, 2004; Luinge and Veltink, 2005).

Foxlin et al. proposed the design of a system consisting of accelerometers and gyroscopes using a complimentary Kalman filter. The rotation was represented by Euler angles. The difference between the estimated orientation obtained from integrating the gyroscope measurements and the elevation and bank angles measured by the accelerometer was fused into the Kalman filter to estimate the orientation error. The system was highly affected by the translational acceleration of the head when the measured accelerations were greatly deviated from gravity. In that case, the Kalman filter incorporated the error in the accelerometer measurements into the estimation of the orientation which led into inaccurate orientation estimates (Foxlin, 1996).

In a later study, Foxlin et al. described a design consisted of two commercially available motion tracking systems composed of accelerometers, gyroscopes and magnetometers for tracking the head. The sensors data were fused into a complementary separatebias Kalman filter in which the computation of the optimum estimate is decoupled from the estimate of the bias (Friedland, 1969). The algorithm was able to correct for the drift 
in the orientation estimates only when the measured acceleration was due to the gravitational acceleration. Therefore, in order to correct for the inertial drift errors, it was required for the head to have stationary periods during its motion (Foxlin, Harrington, and Altshuler, 1998).

In 2006, Sabatini et al. established a quaternion-based extended Kalman filter (EKF) using tri-axis gyroscopes, tri-axis accelerometers and tri-axis magnetometers to measure the orientation. The algorithm used a state augmentation technique in the process model and an adaptive measurement noise covariance matrix to compensate for the deviation from gravity and the local earth's magnetic field strength. The authors reported that the accuracy of the tracker worsened during the dynamic conditions due to the errors in the accelerometer measurements which measured both gravity and body acceleration (Sabatini, 2006).

Yun et al. presented an extended Kalman filter design for the estimation of the orientation of human motion. The system consisted of gyroscopes, accelerometers and magnetometers and the design utilized a simple first-order linear process model. The rotations were represented by quaternions. The filter corrected for the drift in the integration of the angular rates continuously with the assumption of a bounded human limb acceleration. In order to validate the filter performance, a rotary tilt table with two degrees of freedom (elevation and bank angles) was used. The filter performed with a static accuracy of less than 2 degrees and a dynamic accuracy of less than 9 degrees for the bank angle during 2 seconds. However, the authors stated that the filter was not applicable to the applications where accelerations due to forces other than gravity were present for an extended period of time (Yun and Bachmann, 2006).

In a subsequent study in 2008, Yun et al. described an orientation estimation algorithm using only accelerometers and magnetometers. The design used the local magnetic field data only for the calculation of azimuth angle (the compass heading angle). 
The decoupling of accelerometer and magnetometer measurements avoided the error in calculating the elevation and bank angles affected by the magnetic variations. The experimental results showed that the algorithm was able to track the orientation when combined with a low-pass filter for the accelerometer data. Therefore, the system was only applicable for tracking the orientation of a static or slow moving subject (Yun, Bachmann, and McGhee, 2008).

Zhou et al. presented a system for measuring the human arm movements for poststroke home-based rehabilitation purposes. The system integrated the measurements from the tri-axial accelerometers and gyroscopes and used a simulated annealing based optimization technique to provide information on the position of the arm. The tracker was able to accurately estimate the location of the arm; however, the algorithm assumed that the shoulder joint was strictly still during the arm movements with no translational acceleration (Zhou, Hu, and Tao, 2006).

Bergmann et al. developed a system for measuring the anatomical joint angles during the stair climbing with IMU's and compared the performance with the measurements acquired from an optical tracking device. Six IMU's were attached securely to the body segments to ensure that the orientation of the sensors did not change with respect to the body segments. They reported an average root mean square error of 4 to 5 degrees for estimating all the joint angles. The authors stated that the results did not suffer from significant drift problems; however, the designed system was evaluated to track a controlled set of movement (stair climbing) for a brief period of time. Additionally, they indicated that high accelerations could reduce the accuracy of the orientation measurements (Bergmann, Mayagoitia, and Smith, 2009). 


\subsection{Limitations of Previous Studies}

Most of the current practices compute the orientation by integrating the angular velocity obtained from the gyroscopes and fusing the accelerometer and magnetometer measurements into a Kalman filter to correct for the accumulation of error and drift in the estimation of orientation. Various calibration methods are used to improve the sensor performance including Zero Velocity Update (ZUPT), calibrating gyros using a predetermined walking path (Alvarez et al., 2012) and estimating gyro errors by rotating IMU (Li, Fang, and Du, 2012; Abdulrahim et al., 2014).

The majority of the current approaches use zero velocity update as the key calibration method in order to improve the indoor position tracking accuracy. Based on this method, position estimates are accurate when measurements of the accelerometer are not significantly deviated from gravity. ZUPT is applied during the still periods when the measured acceleration is only due to gravity and therefore the velocity measurements are updated as zero. This strategy helps with bounding the error growth of the IMU in between the stops. The limitation of this approach is that the sensors need to be still frequently when the accelerometers can be utilized as inclinometers during the still periods and the gyroscopes measure the rotational rate for a brief duration of time from one still moment to the next. In addition, magnetometers are used to improve the orientation estimates about the vertical axis (heading angle) but the major problem with them is their sensitivity to the magnetic disturbances (Jiménez et al., 2010).

This thesis potentially overcomes some of the limitations of the other approaches regarding the accumulation of error in the estimation of elevation and bank angles. The work introduces a method which incorporates the knowledge of human movement to the tracking algorithm to estimate the orientation over a persistent motion and extended period of time using inertial sensors. 


\section{Tracker Design}

The goal of this chapter is to provide a description of the process and measurement models as well as the filter designed to estimate the orientation of the human motion. Section 3.1 provides a background on the Bayesian framework and Bayesian approach to solve the estimation problems. Section 3.2 describes the nonlinear state space models as a common approach to tracking and navigation problems. Section 3.3 discusses the dynamic filters such as particle filtering and variants of the Kalman filtering to estimate time-varying states. Section 3.4 describes the process and the measurement models designed in this work as well as introduces the concepts of the quaternions and the Euler angles as two common approaches of the rotation representation which have been utilized in this thesis. The last section, 3.5, introduces the designed UKF applied in this work.

\subsection{Bayesian Framework}

Probabilistic inference refers to the process of estimating hidden states which are caused by noisy or incomplete measurements. The common classical estimators are arithmetic, geometric or harmonic mean, median, mode and root mean square. Despite classical estimation approaches which assume that the parameter of interest which we want to estimate is unknown and deterministic, Bayesian approach assumes that the estimated variable, $\mathbf{x}$, is random and unknown. The estimators are also random variables, $\hat{\mathbf{x}}$, and a

function of the measurements $\hat{\mathbf{x}}\left[\left.\left\{\mathbf{y}_{n}\right\}\right|_{1} ^{N}\right]$; therefore, the estimator varies based on the set of observations. In Bayesian approach, the random variable $\mathbf{x}$ varies for each realization 
and changes over the ensemble. Bayesian estimation allows the incorporation of the available prior knowledge as a prior Probability Density Function (PDF) of $\mathbf{x}$. At least, it can improve the accuracy, when the prior knowledge is both accurate and useful.

The Bayes' theorem states:

$$
p(\mathbf{x}, \mathbf{y})=p(\mathbf{x} \mid \mathbf{y}) p(\mathbf{y})=p(\mathbf{y} \mid \mathbf{x}) p(\mathbf{x})
$$

And the Bayesian mean square error is obtained from the following equation:

$$
\operatorname{MSE}_{B}(\hat{\mathbf{x}})=\iint(\mathbf{x}-\hat{\mathbf{x}})^{2} p(\mathbf{y}, \mathbf{x}) d y d x
$$

The mean of the posterior PDF, $p(\mathbf{x} \mid \mathbf{y})$, is an optimal estimator that minimizes the Bayesian MSE and therefore, its distribution is of significant importance in estimating $\mathbf{x}:$

$$
\hat{\mathbf{x}}=E[\mathbf{x} \mid \mathbf{y}]=\int \mathbf{x} p(\mathbf{x} \mid \mathbf{y}) d x
$$

In the Bayesian approach, the assignment of a prior PDF is required, and if the information is unavailable, the prior PDF is presented as a uniform prior over a wide range. After acquiring the measurement data, the knowledge is summarized by the posterior PDF of $\mathbf{x}$. The Bayesian estimator relies on both the prior knowledge as well as the measured data; therefore, the question of how to properly justify the given weight to the prior knowledge plays an important role in the Bayesian estimation. The state space tracking methods rely on the prior knowledge determined by the physical constraints or obtained from the past observations (Arulampalam et al., 2002, Gordon, Ristic, and Arulampalam, 2004, Kailath, Sayed, and Hassibi, 2000). 


\subsection{Nonlinear State Space Model}

State space models are common approaches to tracking and navigation problems. A state space model can describe a stochastic, time-varying, nonlinear process by a vector of quantities known as the state vector, $\mathbf{x}_{n}$ in which the subscript $n$ is the index for the discrete time. The state vector is the smallest $m$-dimensional vector which fully describes the past of a deterministic dynamic system. The state is estimated based on the prior knowledge about the dynamics of the system combined with the acquired finite sets of the measurements. The states trace the behavior of an object in time and the state space includes all the possible states. The trajectory of an object in the state space describes how the states transition sequentially over time (Kay, 1993). The observed measurement is a function of time represented by an $k$-dimensional vector, $\mathbf{y}_{n}$. Equations 3.4 and 3.5 are the general representation of a state space model.

$$
\begin{array}{r}
\mathbf{x}_{n+1}=f_{n}\left(\mathbf{x}_{n}, \mathbf{w}_{n}\right) \\
\mathbf{y}_{n}=h_{n}\left(\mathbf{x}_{n}, \mathbf{v}_{n}\right)
\end{array}
$$

where $f_{n}($.$) and h_{n}($.$) are nonlinear models describing the process and measurement$ models respectively, and $\mathbf{w}_{n}$ and $\mathbf{v}_{n}$ are uncorrelated random processes representing the process and measurement noise which are white noise with zero means. In addition, the state space models are Markov processes which implies that the output at any stage depends only on the output of the previous stage; therefore, if we have information about the present state at time $n$, then the previous values of the state, $N=0: n-1$, would not provide any additional information in order to predict the state at time $n+1$. 


$$
\begin{gathered}
p\left(\mathbf{y}_{n} \mid \mathbf{x}_{0: n}\right)=p\left(\mathbf{y}_{n} \mid \mathbf{x}_{n}\right) \\
p\left(\mathbf{x}_{n+1} \mid \mathbf{x}_{0: n}\right)=p\left(\mathbf{x}_{n+1} \mid \mathbf{x}_{n}\right)
\end{gathered}
$$

The primary goal of a state space tracking problem is to estimate the state of the system, $\mathbf{x}_{n}$, at time $n$ given a sequence of observations, $\left.\left\{\mathbf{y}_{n}\right\}\right|_{0} ^{N-1}$. In the context of state space tracking, the Bayes' theorem becomes:

$$
p\left(\mathbf{x}_{0: n}, \mathbf{y}_{n} \mid \mathbf{y}_{0: n-1}\right)=p\left(\mathbf{x}_{0: n} \mid \mathbf{y}_{n}, \mathbf{y}_{0: n-1}\right) p\left(\mathbf{y}_{n} \mid \mathbf{y}_{0: n-1}\right)=p\left(\mathbf{x}_{0: n} \mid \mathbf{y}_{0: n}\right) p\left(\mathbf{y}_{n} \mid \mathbf{y}_{0: n-1}\right)
$$

In order to construct the PDF $p\left(\mathbf{x}_{n} \mid \mathbf{y}_{0: n}\right)$, it is assumed that the prior PDF $p\left(\mathbf{x}_{0} \mid \mathbf{y}_{0}\right) \equiv$ $p\left(\mathbf{x}_{0}\right)$ is available. Therefore, it is feasible to compute the PDF $p\left(\mathbf{x}_{n} \mid \mathbf{y}_{0: n}\right)$ recursively using two steps of prediction and update. The prediction step utilizes the ChapmanKolmogorov equation, 3.9, to obtain the prior PDF of $\mathbf{x}_{n}$ :

$$
p\left(\mathbf{x}_{n} \mid \mathbf{y}_{0: n-1}\right)=\int p\left(\mathbf{x}_{n} \mid \mathbf{x}_{n-1}\right) p\left(\mathbf{x}_{n-1} \mid \mathbf{y}_{0: n-1}\right) d \mathbf{x}_{n-1}
$$

As the measurement $\mathbf{y}_{n}$ is available at time $n$, the prior PDF becomes updated (update step) using the Bayes' theorem, 3.10:

$$
p\left(\mathbf{x}_{n} \mid \mathbf{y}_{0: n}\right)=\frac{p\left(\mathbf{y}_{n} \mid \mathbf{x}_{n}\right) p\left(\mathbf{x}_{n} \mid \mathbf{y}_{0: n-1}\right)}{p\left(\mathbf{y}_{n} \mid \mathbf{y}_{0: n-1}\right)}
$$

Evaluation of the above recursive equations is impractical particularly for higher dimensional spaces. Particle filtering and variations of Kalman filtering are the two common approaches to address this issue and determine the posterior density (Kay, 1993, Arulampalam et al., 2002). 


\subsection{Estimation and Filtering}

As it was discussed in the previous chapter, the estimation of orientation with IMU's is feasible by fusing the measurements from the auxiliary sensors into a complementary filter to confine the orientation drift error from the gyroscope measurements using a process model which describes the dynamics of the human movement. If the process model can be described by discrete-time-varying states then the states can be sequentially estimated by probabilistic Bayesian approaches (Lim, Shin, and Hwang, 2017). The time-varying states are estimated using sensor measurements at each time step and dynamic filters such as particle filtering and Kalman filtering.

Particle filters are one of the common approaches of solving for the state space models. The particle filtering approach approximates the marginal distribution of the hidden state using the measurement data and assuming that the posterior is represented as the sum of multi-dimensional impulses. At each sample point, the distribution is approximated with a weighted set of discrete values, known as particles, using sequential importance sampling (SIS) (Gordon, Ristic, and Arulampalam, 2004). Particle filters are easy to implement; however, the computational demands of particle filters tend to be quite high. Additionally, particle filters don't work well if there is no process or measurement noise in the system (Arulampalam et al., 2002).

\subsubsection{Kalman Filter and Its Variations}

The Kalman filter assumes that the process and the measurement models, $f_{n}($.$) and$ $h_{n}($.$) , are linear functions, and the additive noise, \mathbf{w}_{n}$ and $\mathbf{v}_{n}$, are white noise but not necessarily Gaussian. If the two aforementioned conditions are met, the Kalman filter minimizes the mean square error of the estimated parameters (Arulampalam et al., 2002). However, most of the real world problems are nonlinear which significantly limits the application of the Kalman filter. 
The extended Kalman filter (EKF) is an extension of the standard Kalman filter which uses Taylor series to linearize a nonlinear model. Although the EKF is widely used for solving the nonlinear problems, it is not optimal for highly nonlinear problems due to the low stability and computational tractability (Lim, Shin, and Hwang, 2017).

The unscented Kalman filter (UKF) is another variation of the Kalman filter which estimates the propagation of the state variable distribution more accurately for highly nonlinear models. The UKF is also known as the sigma-point Kalman filter since it uses a set of properly selected samples so-called sigma points which represent the mean and the variance of the state distribution for the nonlinear models. The UKF is more computationally tractable and stable in the convergence compared to the EKF. They also don't require the computation of the Jacobian matrices which is highly time consuming and prone to error (Lim, Shin, and Hwang, 2017).

\subsection{Process and Measurement Models}

The design of the process model and the measurement model is required to estimate the future states of a dynamic system. The process model describes the evolution of the state over time and the measurement model relates the noisy measurements to the state (Arulampalam et al., 2002). In this work, the goal was to estimate the orientation represented by the quaternions, $\mathbf{q}_{n}$, given a sequence of measured accelerations and rotational rates from 0 to $n-1,\left.\mathbf{a}_{n}\right|_{0} ^{n-1}$ and $\left.\boldsymbol{\omega}_{n}\right|_{0} ^{n-1}$, respectively.

\subsubsection{Quaternions}

The orientation quaternion was used in this work to represent the orientation of the human body in space. A quaternion is a 4-tuple of real numbers:

$$
\mathbf{q}=\left(q_{0}, q_{1}, q_{2}, q_{3}\right)
$$


An alternative way of representing a quaternion is as the sum of a scalar, $q_{0}$, and a vector, $\left(q_{1}, q_{2}, q_{3}\right)$ :

$$
\mathbf{q}=q_{0}+\mathbf{i} q_{1}+\mathbf{j} q_{2}+\mathbf{k} q_{3}
$$

This alternative form of representation allows the quaternions to play an important role in representing a rotation operator where they are interpreted geometrically as a rotation in $R^{3}$ by specifying the angle and the axis of rotation.

\subsubsection{Quaternion Rotation Operator}

Before introducing the quaternions as the rotation operators, it is required for the reader to be familiar with some of the quaternion algebra such as addition, multiplication and complex conjugate.

The addition of two quaternions $\mathbf{p}$ and $\mathbf{q}$ is defined as the sum of the corresponding components:

$$
\mathbf{p}+\mathbf{q}=\left(p_{0}+q_{0}\right)+\mathbf{i}\left(p_{1}+q_{1}\right)+\mathbf{j}\left(p_{2}+q_{2}\right)+\mathbf{k}\left(p_{3}+q_{3}\right) .
$$

The product of two quaternions $\mathbf{p}$ and $\mathbf{q}$ is defined as follows:

$\mathbf{p} \mathbf{q}=p_{0} q_{0}-\left(p_{1}, p_{2}, p_{3}\right) \cdot\left(q_{1}, q_{2}, q_{3}\right)+p_{0}\left(q_{1}, q_{2}, q_{3}\right)+q_{0}\left(p_{1}, p_{2}, p_{3}\right)+\left(p_{1}, p_{2}, p_{3}\right) \times\left(q_{1}, q_{2}, q_{3}\right)$

where and $\times$ are the dot product and the cross product of the vector parts of the quaternions respectively.

The complex conjugate of the quaternion $\mathbf{q}$ is defined as:

$$
\mathbf{q}^{*}=q_{0}-\mathbf{i} q_{1}-\mathbf{j} q_{2}-\mathbf{k} q_{3}
$$


The quaternion rotation operator, $L_{\mathbf{q}}$, can be considered as a triple quaternion product defined as follows:

$$
L_{\mathbf{q}}(\mathbf{v})=\mathbf{q} \mathbf{v} \mathbf{q}^{*}
$$

where $\mathbf{q}$ is a unit or normalized quaternion represented as:

$$
\mathbf{q}=\cos (\theta)+\mathbf{u} \sin (\theta)
$$

and the vector $\mathbf{v}$ is considered as a pure quaternion whose scalar part is zero, $\mathbf{v}=0+\mathbf{v}$. Therefore, $L_{\mathbf{q}}(\mathbf{v})$ can be interpreted geometrically as a rotation of the vector $\mathbf{v}$ through an angle $2 \theta$ about the axis of rotation represented by $\mathbf{u}$. A sequence of rotations are represented by the quaternion product $\mathbf{q p}$ which indicates a rotation operator $L_{\mathbf{p}}$ followed by $L_{\mathbf{q}}$ (Kuipers, 1999).

\subsubsection{Euler Angles}

Euler angles are another way to describe an orientation of a rigid body with respect to a fixed coordinate system. They represent a sequence of the angles of rotation about a coordinate axis. In this work, the Euler angles are shown as heading $(\psi)$, elevation $(\theta)$ and bank $(\phi)$ angles. The heading indicates the rotation about the Z-axis, the elevation specifies the rotation about the Y-axis and the bank demonstrates the rotation about the X-axis. There are at least twelve Euler angle-axis sequences which can relate any two arbitrary but distinct coordinate frames. These sequences include: $x y z, x z y, x y x$, $x z x, y z x, y x z, y z y, y x y, z x y, z y x, z x z, z y z$. For instance, the sequence xyz represents a rotation about the $\mathrm{X}$-axis, followed by a rotation about the new $\mathrm{y}$-axis, followed by a rotation about the newer z-axis (Kuipers, 1999).

In this thesis, the orientation quaternions were used to represent the orientation due to some of the advantages of quaternions over the Euler angles and the rotation 
matrices. Some of these advantages include:

- Quaternions have a more obvious geometrical interpretation compared to the Euler angles

- They are independent of the choice of the coordinate system

- Quaternions allow using a simpler method of interpolation than Euler angles

- They are a more compact representation of rotation compared to the rotation matrices

- Quaternions do not suffer from gimbal lock (Dam, Koch, and Lillholm, 1998)

\subsubsection{Process Model}

In this thesis, the state vector consists of the rotation quaternion, $\mathbf{q}$, augmented by the estimated translational velocity, $\mathbf{v}$, and position, p. Equation 3.18 describes the process model at time $n+1$ while the user has to provide the initial state. The orientation at time $n+1$ is defined as the quaternion product of the two quaternions $\mathbf{q}_{n}$ and $\mathbf{r}_{n}$ representing the rotation $\mathbf{q}_{n}$ followed by the rotation $\mathbf{r}_{n}$ at time $n$.

$$
\mathbf{x}_{n+1}=\left(\begin{array}{c}
\mathbf{q}_{n+1} \\
\mathbf{v}_{n+1} \\
\mathbf{p}_{n+1}
\end{array}\right)=\left(\begin{array}{c}
\mathbf{r}_{n} \mathbf{q}_{n} \\
\mathbf{v}_{n}+\frac{1}{f_{s}}\left(C\left(\mathbf{q}_{n}\right) \mathbf{a}_{n}-\mathbf{g}\right) \\
\mathbf{p}_{n}+\frac{1}{f_{s}} \mathbf{v}_{n}
\end{array}\right)+\left(\begin{array}{c}
\mathbf{w}_{\mathbf{q}, n} \\
\mathbf{w}_{\mathbf{v}, n} \\
\mathbf{w}_{\mathbf{p}, n}
\end{array}\right)
$$

The quaternion $\mathbf{r}_{n}$ is obtained from the gyroscope measurements using equations 3.19 - 3.21. The gyroscope measures the angular velocity, $\boldsymbol{\omega}_{n}$, at a sample rate of $f_{s}$ and the angles $\left(\theta_{n}, \phi_{n}, \psi_{n}\right)$ specify the Euler angles sequence at time $n$. The rotation quaternion $\mathbf{r}_{n}$ indicates a rotation with an angle of $\frac{\Phi_{n}}{2}$ about an axis of rotation represented by its vector part. 


$$
\begin{aligned}
\left(\theta_{n}, \phi_{n}, \psi_{n}\right) & =\frac{1}{f_{s}} \boldsymbol{\omega}_{n} \\
\Phi_{n} & =\left\|\left(\theta_{n}, \phi_{n}, \psi_{n}\right)\right\| \\
\mathbf{r}_{n} & =\cos \left(\frac{\Phi_{n}}{2}\right)+\frac{1}{\Phi_{n}} \sin \left(\frac{\Phi_{n}}{2}\right)\left(\theta_{n}, \phi_{n}, \psi_{n}\right)
\end{aligned}
$$

The linear velocity, $\mathbf{v}_{n+1}$, at time $n+1$ is obtained using the forward recursive Newton-Euler equation of motion in equation 3.22. The accelerometer measures both the translational acceleration of the sensor as well as the contribution of the normal forces that prevent the sensor from accelerating toward the center of the Earth (Robotics, 2012). The rotational matrix $C\left(\mathbf{q}_{n}\right)$, a function of $\mathbf{q}_{n}$, converts the measured acceleration, $\mathbf{a}_{n}$, from the the body frame of the sensor to the Earth inertial frame and $\mathbf{g}$ is the force of gravity equivalent to $-9.81 \frac{\mathrm{m}}{\mathrm{s}^{2}}$.

$$
\mathbf{v}_{n+1}=\mathbf{v}_{n}+\frac{1}{f_{s}}\left(C\left(\mathbf{q}_{n}\right) \mathbf{a}_{n}-\mathbf{g}\right)
$$

The linear position, $\mathbf{p}_{n+1}$, at time $n+1$ is computed using the forward recursive Newton-Euler equation of motion stated by equation 3.23.

$$
\mathbf{p}_{n+1}=\mathbf{p}_{n}+\frac{1}{f_{s}} \mathbf{v}_{n}
$$

\subsubsection{Measurement Model}

In general measurement model maps the states to the measurement data. In this design, the measurement model uses the process model, $f_{n}$, and the measurements from the accelerometer and the gyroscope to estimate the position $N$ samples or equivalently $T$ seconds in the future where $T=N \frac{1}{f_{s}}$. Equation 3.24 describes the measurement model using a for loop in which the state at time $n+N$ is estimated. Equation 3.25 is a more 
compact representation of the measurement model where the operator $f^{N}$ indicates that the measurement model applies to the process model $N$ times.

$$
\begin{aligned}
& \mathbf{y}_{n+N}=f\left(f\left(f\left(\ldots f\left(f\left(\mathbf{y}_{n}, \boldsymbol{\omega}_{n}\right), \boldsymbol{\omega}_{n+1}\right) \ldots\right), \boldsymbol{\omega}_{n+N-2}\right), \boldsymbol{\omega}_{n+N-1}\right) \\
& \mathbf{y}_{n+N}=f^{N}\left(\mathbf{y}_{n}, \boldsymbol{\omega}_{n: n+N}\right)
\end{aligned}
$$

The vector in Equation 3.26, $\mathbf{y}_{n+N}$, is the estimated state vector, $\mathbf{x}_{n+N}$, at time $n+N$ used in the computation of the measurement vector, Equation 3.27.

$$
\mathbf{y}_{n+N}=\mathbf{x}_{n+N}=\left(\begin{array}{c}
\mathbf{q}_{n+N} \\
\mathbf{v}_{n+N} \\
\mathbf{p}_{n+N}
\end{array}\right)
$$

The output of the measurement model is the estimated position, $\mathbf{p}_{n+N}$, at time $n+N$ which corresponds to the last three elements of $\mathbf{y}_{n+N}$ :

$$
\mathbf{p}_{n+N+1}=\mathbf{p}_{n+N}+\frac{1}{f_{s}} \mathbf{v}_{n+N}
$$

The position can be simply estimated by double integrating the measurements from the accelerometer; however, as it was discussed in the previous chapters, the main problem with this approach is the accumulation of error in the position estimation which grows cubically in time when integrating the noisy acceleration measurements (Foxlin, 2005). The measurement model in this design utilizes the prior knowledge about the natural range of human movement in order to confine the unbounded growth of position estimation when double integrating the acceleration. It is evident that humans cannot move naturally more than few meters during $1 s$ time period; therefore, this information can be used to prevent the unconstrained growth of the position. The designed measurement model performs this task by calculating the position for $T s$ in 
the future using the process model and integrating this knowledge into the UKF. This approach potentially decreases the uncertainty in using the measurement model in the UKF even during the persistent motions when the accelerometer measurements are significantly deviated from gravity.

\subsection{Filter Design}

In this work, the UKF was implemented as the tracking algorithm to estimate the orientation. The UKF estimates the propagation of the state variable distribution more accurately for highly nonlinear models compared to the EKF. Using the UKF, it is feasible to calculate the posterior mean and covariance of the state accurately up to the third order for any nonlinearity. In addition, the UKF does not require the calculation of the Jacobian matrices which can be non-trivial and result in the implementation difficulties (Lim, Shin, and Hwang, 2017, Wan and Van Der Merwe, 2000).

The UKF uses $2 L$ sigma points where $L$ is the dimension of the state, $\mathbf{x}_{n}$, and the sigma points are a minimal set of carefully chosen sample points which describe the mean and covariance of the previous state. The sigma points, $\mathbf{x}_{n}{ }^{i}$, are calculated using equations $3.28-3.30$ for $i=1, \ldots, 2 L$

$$
\mathbf{x}_{n}{ }^{i}=\hat{\mathbf{x}}_{n}+\tilde{\mathbf{x}}^{i}
$$

for $i=1, \ldots, L$

$$
\tilde{\mathbf{x}}_{n}^{i}=\left[\operatorname{row}_{i}\left(\sqrt{L P_{n}}\right)\right]^{T}
$$

for $i=L+1, \ldots, 2 L$

$$
\tilde{\mathbf{x}}_{n}^{i}=-\left[\operatorname{row}_{i}\left(\sqrt{L P_{n}}\right)\right]^{T}
$$


where $\hat{\mathbf{x}}_{n}=\frac{1}{2 L} \sum_{i=1}^{2 L} \mathbf{x}_{n}{ }^{i}$ and $P_{n}=\frac{1}{2 L} \sum_{i=1}^{2 L}\left(\mathbf{x}_{n}{ }^{i}-\hat{\mathbf{x}}_{n}\right)\left(\mathbf{x}_{n}{ }^{i}-\hat{\mathbf{x}}_{n}\right)^{T}$ are the mean and covariance of the state at time $n$ respectively. Equations 3.31-3.34 indicate the prediction step or the time update step:

$$
\begin{aligned}
\mathbf{x}_{n-1}^{i} & =\hat{\mathbf{x}}_{n-1 \mid n-1}+\tilde{\mathbf{x}}^{i} \\
\hat{\mathbf{x}}_{n}^{i} & =f_{n}\left(\hat{\mathbf{x}}_{n-1}^{i}\right)+Q_{n-1} \\
\hat{\mathbf{x}}_{n \mid n-1} & =\frac{1}{2 L} \sum_{i=1}^{2 L} \hat{\mathbf{x}}_{n}^{i} \\
P_{\tilde{\mathbf{x}}, n \mid n-1} & =\frac{1}{2 L} \sum_{i=1}^{2 L}\left(\mathbf{x}_{n}{ }^{i}-\hat{\mathbf{x}}_{n \mid n-1}\right)\left(\mathbf{x}_{n}{ }^{i}-\hat{\mathbf{x}}_{n \mid n-1}\right)^{T}
\end{aligned}
$$

The filter step or the measurement-update equations are calculated using equations 3.35-3.41:

$$
\begin{aligned}
\mathbf{y}_{n}^{i} & =h_{n}\left(\mathbf{x}_{n}{ }^{i}\right) \\
\hat{\mathbf{y}}_{n \mid n-1} & =\frac{1}{2 L} \sum_{i=1}^{2 L} \mathbf{y}_{n}{ }^{i} \\
P_{\tilde{y}} & =\frac{1}{2 L} \sum_{i=1}^{2 L}\left(\mathbf{y}_{n}{ }^{i}-\hat{\mathbf{y}}_{n \mid n-1}\right)\left(\mathbf{y}_{n}{ }^{i}-\hat{\mathbf{y}}_{n \mid n-1}\right)^{T}+R_{n-1} \\
P_{\tilde{x} \tilde{y}} & =\frac{1}{2 L} \sum_{i=1}^{2 L}\left(\mathbf{x}_{n}{ }^{i}-\hat{\mathbf{x}}_{n \mid n-1}\right)\left(\mathbf{y}_{n}{ }^{i}-\hat{\mathbf{y}}_{n \mid n-1}\right)^{T} \\
K_{n} & =P_{\tilde{\mathbf{x}} \tilde{y}} P_{\tilde{y}}{ }^{-1} \\
\hat{\mathbf{x}}_{n \mid n} & =\hat{\mathbf{x}}_{n \mid n-1}+K_{n}\left(\mathbf{y}_{n}-\hat{y}_{n \mid n-1}\right) \\
P_{\tilde{\mathbf{x}}, n \mid n} & =P_{\tilde{\mathbf{x}}, n \mid n-1}-K_{n} P_{\tilde{y}} K_{n}{ }^{T}
\end{aligned}
$$

where $K_{n}$ is the Kalman gain and $P_{\tilde{\mathbf{x}}, n \mid n}$ represents the updated state covariance (Simon, 2006). 


\subsubsection{UKF Design Parameters}

The process and measurement noise covariances, the initial state estimate and its corresponding covariance are the design parameters of the UKF whose proper selections would affect the performance of the tracking algorithm.

The $Q$ and $R$ matrices represent the process and the measurement noise covariances respectively. The $R$ matrix was determined empirically representing the uncertainty in the natural range of human movement, $\sigma_{R}$. In this work, the measurement noise covariance was assumed diagonal and time-invariant, and $\sigma_{R}$ was approximated as $10 \mathrm{~m}$ reflecting the natural human motion displacement during a $1 \mathrm{~s}$ time interval (Equation 3.42).

$$
R=\operatorname{diag}\left(\sigma_{R}^{2}\right)
$$

The $Q$ matrix describes the covariance of the additive noise to the process model, $\mathbf{w}_{n}$, which consists of three elements: the noise on the quaternion, velocity and position parts of the state vector represented by $\mathbf{w}_{\mathbf{q}, n}, \mathbf{w}_{\mathbf{v}, n}$ and $\mathbf{w}_{\mathbf{p}, n}$ respectively. The standard

deviation of the noise on the velocity and position, $\mathbf{w}_{\mathbf{v}, n}$ and $\mathbf{w}_{\mathbf{p}, n}$ were estimated using Equations 3.43 and 3.44 over 1000 realizations. Equation 3.43 assumes that the single source of error when estimating the velocity originates from the noise on the accelerometer measurements and the fact that the acceleration is not constant over one sampling interval. Equation 3.44 estimates the standard deviation of the noise on the position, $\mathbf{w}_{\mathbf{p}, n}$, with the assumption that the error originates from the noise on the velocity.

$$
\begin{aligned}
& \mathbf{w}_{\mathbf{v}, n}=\mathbf{v}_{n+1}-\left(\mathbf{v}_{n}+\frac{1}{f_{s}}\left(C\left(\mathbf{q}_{n}\right) \mathbf{a}_{n}-\mathbf{g}\right)\right) \\
& \mathbf{w}_{\mathbf{p}, n}=\mathbf{p}_{n+1}-\left(\mathbf{p}_{n}+\frac{1}{f_{s}} \mathbf{v}_{n}\right)
\end{aligned}
$$


The noise on the quaternion part of the state vector, $\mathbf{w}_{\mathbf{q}, n}$, was considered as:

$$
\mathbf{w}_{\mathbf{q}, n}=\mathbf{q}_{n+1}-\mathbf{r}_{n} \mathbf{q}_{n}
$$

which is the difference between the true quaternion at time $n+1$ and the quaternion obtained from the quaternion product of $\mathbf{q}_{n}$ and $\mathbf{r}_{n}$. When estimating $\mathbf{w}_{\mathbf{q}, n}$, it was assumed that the noise on the gyroscope measurements was the single source of creating the noise. The noise was estimated as the average of 1000 realizations using Equation 3.45 .

The process noise covariance was designed as a constant and diagonal matrix. The diagonal property of the matrix indicates that the individual components of $\mathbf{w}_{n}$, i.e., $\mathbf{w}_{\mathbf{q}, n}, \mathbf{w}_{\mathbf{v}, n}$ and $\mathbf{w}_{\mathbf{p}, n}$ are uncorrelated. Equation 3.46 defines the process noise covariance where ${\sigma_{\mathbf{q}}}^{2}, \sigma_{\mathbf{v}}{ }^{2}$ and ${\sigma_{\mathbf{p}}}^{2}$ are the variances of $\mathbf{w}_{\mathbf{q}, n}, \mathbf{w}_{\mathbf{v}, n}$ and $\mathbf{w}_{\mathbf{p}, n}$ respectively.

$$
Q=\operatorname{diag}\left(\left[\begin{array}{ccc}
\sigma_{\mathbf{q}}^{2} & \sigma_{\mathbf{v}}^{2} & \sigma_{\mathbf{p}}{ }^{2}
\end{array}\right]\right)
$$

The diagonal elements of $Q$ and $R$ are used as the tuning parameters of the algorithm. They determine how much the Kalman filter relies on the process and the measurement model. The higher the measurement noise covariance, the Kalman filter relies more heavily on the process model; whereas, the lower the measurement noise covariance, the Kalman filter more confidently uses the measurement model. 


\begin{tabular}{|c|c|c|}
\hline \multicolumn{3}{|c|}{ User-Specified Parameters } \\
\hline Variable Name & Symbol & Value \\
\hline $\begin{array}{l}\text { Standard deviation of process noise (quater- } \\
\text { nion part) }\end{array}$ & $\sigma_{\mathbf{q}}$ & $2 \times 10^{-3}$ a.u. \\
\hline $\begin{array}{l}\text { Standard deviation of process noise (velocity } \\
\text { part) }\end{array}$ & $\sigma_{\mathbf{v}}$ & $5 \times 10^{-3} \frac{\mathrm{m}}{\mathrm{s}}$ \\
\hline $\begin{array}{l}\text { Standard deviation of process noise (position } \\
\text { part) }\end{array}$ & $\sigma_{\mathbf{p}}$ & $5 \times 10^{-3} \mathrm{~m}$ \\
\hline Standard deviation of measurement noise & $\sigma_{R}$ & $\begin{array}{l}10 \mathrm{~m} \\
(1)\end{array}$ \\
\hline Initial state (quaternion part) & $\mathbf{q}_{0}$ & $\begin{array}{l}0 \\
0\end{array}$ \\
\hline Initial state (velocity part) & $\mathbf{v}_{0}$ & \begin{tabular}{l|l}
0 & $\frac{\mathrm{m}}{\mathrm{s}}$ \\
0 &
\end{tabular} \\
\hline Initial state (position part) & $\mathbf{p}_{0}$ & 0 \\
\hline $\begin{array}{l}\text { Standard deviation of initial state (quaternion } \\
\text { part) }\end{array}$ & $\sigma_{\mathbf{x}_{0}}$ & $10^{-4}$ a.u. \\
\hline $\begin{array}{l}\text { Standard deviation of initial state (velocity } \\
\text { part) }\end{array}$ & $\sigma_{\mathbf{x}_{0}}$ & $10^{-4} \frac{\mathrm{m}}{\mathrm{s}}$ \\
\hline $\begin{array}{l}\text { Standard deviation of initial state (position } \\
\text { part) }\end{array}$ & $\sigma_{\mathbf{x}_{0}}$ & $10^{-4} \mathrm{~m}$ \\
\hline
\end{tabular}

TABLE 3.1: User-specified parameters of UKF design 


\section{Performance Assessment}

The goal of this chapter is to assess the performance of the proposed tracking method, short-term tracking algorithm, through two approaches: applying the algorithm to a synthetic data and a real data. The synthetic data allows one to inexpensively create a long duration data while it is costly to obtain long recordings of real data. In addition, with the synthetic data the user has more control on designing the statistical properties of the movement such as the speed and type of motion while this is not easily available when working with the real data. In this work, the synthetic data was designed as a random process to simulate the human motion while having control on designing its statistical properties, and the real data was collected using an IMU and a 6-axis high precision industrial robot arm.

\subsection{Model Validation Using Synthetic Data}

Despite the complexity of the human movement and the unique characteristics of the movement of the different parts of the body, there are certain properties which are common throughout the motion regardless of the location of the movement on the body. These common properties include the confinement of the motion. Humans tend to restrict their movement in a limited space; thus, certain properties of their motion such 
as position, velocity and acceleration are confined within boundaries and do not grow unboundedly. Therefore, it was reasonable to model the human motion as the changes in the position and rotation of one point of the movement. The synthetic data was designed with the necessary requirement of having the position, velocity and acceleration of the point of the human movement bounded.

\subsubsection{Design of Synthetic Data}

This section describes the design goals and decisions involved in the design of the stochastic model representing the human movement.

\section{Design Goals for Modeling Human Motion}

1. Simplicity: Simplicity was one of the design goals in this thesis while capturing the relevant complexities of the human motion.

2. Compliancy with the Newton's second law of motion: The law states that the acceleration of a mass is proportional to the amount of the force applied to it. It is known that the position and velocity of human movement do not change instantaneously but they change over time as a result of the exertion of the force or torque. Equivalently, the position and velocity signals of human movement contain higher power for the low frequency components. However, the acceleration of the human motion which is equivalent to the force exerted by the joints and muscles can change more rapidly compared to the changes in the position 
and velocity; therefore, the acceleration signal of the human movement has more power for the high frequency components. The computation of the velocity and position through integrating the acceleration once and twice respectively will result in producing low-frequency signals since the integration operation is a form of low-pass filtering.

3. Wide-sense stationarity: It is known that the translational acceleration, velocity and position as well as the angular velocity of human movement are persistent and do not change over time significantly. In addition, the variance of these signals is constant over an observed period of time. Therefore, the essential properties of human movement can be modeled as a wide-sense stationary (WSS) over the time interval the signals are observed. A WSS signal has constant mean and variance and its autocorrelation is a function of the time difference between the samples.

4. The range of the standard deviation of human movement properties: The standard deviations of the translational acceleration, $\sigma_{\mathbf{a}}$, velocity, $\sigma_{\mathbf{v}}$, and position, $\sigma_{\mathbf{p}}$, as well as the angular velocity, $\sigma_{\boldsymbol{\omega}}$, of human movement in an indoor space such as a house or office doing regular daily activities are confined and do not grow unboundedly. The standard deviation of the acceleration of the indoor human movement is within the range of $\pm 5 g$ where $g$ is the gravitational force equivalent to $9.81 \frac{\mathrm{m}}{\mathrm{s}^{2}}$, and the standard deviations of the translational position, velocity 
and angular velocity of the described movement are within $\pm 10 \mathrm{~m}, \pm 5 \mathrm{~m} / \mathrm{s}$ and $100-200^{\circ} / \mathrm{s}$ respectively.

\section{Design Decisions for Modeling Human Motion}

1. Design of translational acceleration, velocity and position: White noise is the simplest form of the stochastic processes which has the same amount of power for all the frequencies. The Gaussian distribution of white noise is significantly important because of the central limit theorem which states that in general the sum of an infinite number of statistically independent random variables has Gaussian distribution. The simplest method to model the acceleration signal, $\mathbf{a}_{n}$, was to apply a high-pass filter to white noise, $\mathbf{w}_{n}$, with zero mean and Gaussian distribution. Figure 4.1 demonstrates the block diagram of the design of the translational acceleration, velocity and position with the knowledge that the integration process involved in creating the velocity and position is a form of low-pass filtering.

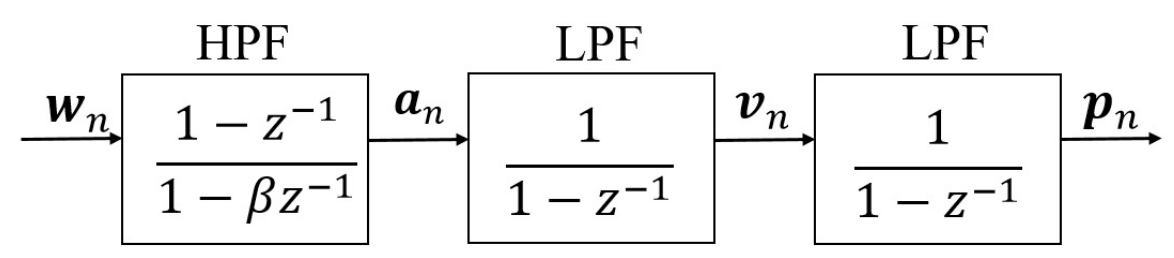

FIGURE 4.1: Block diagram of design of human movement properties

2. Design of high-pass filter: A high-pass filter enforces zero gain at zero frequency which is also known as the DC frequency. A high-pass filter with two zeros at 
the DC frequency was applied to $\mathbf{a}_{n}$ to ensure that when integrating the acceleration once and twice to create the velocity, $\mathbf{v}_{n}$, and position, $\mathbf{p}_{n}$, they were both bounded. The simplest method of designing a high-pass filter which would satisfy the design requirements was a second order filter. For the simplicity purposes, a first order non-causal infinite impulse response (IIR) filter was designed which filtered the signal both in the forward and backward directions. The two zero's of the high-pass filter were placed on $(1,0)$ of the unit circle which is equivalent to zero frequency to ensure zero magnitude response at zero frequency. The choice of the pole of the filter, $\beta$, which determines the filter cutoff frequency, affected the distribution of the power of the acceleration signal. Equation 4.1 is the transfer function of the designed high-pass filter.

$$
H(z)=\frac{1-z^{-1}}{1-\beta z^{-1}}
$$

where $\beta$ was chosen as 0.9986 in order to satisfy the fourth design goal regarding the range of the standard deviation of the motion translational properties. Figures 4.2 and 4.3 demonstrate that the greater values of $\beta$ led into larger, $\sigma_{\mathbf{v}}$, and, $\sigma_{\mathbf{p}}$, while the smaller values of $\beta$ decreased the velocity and position standard deviations. 


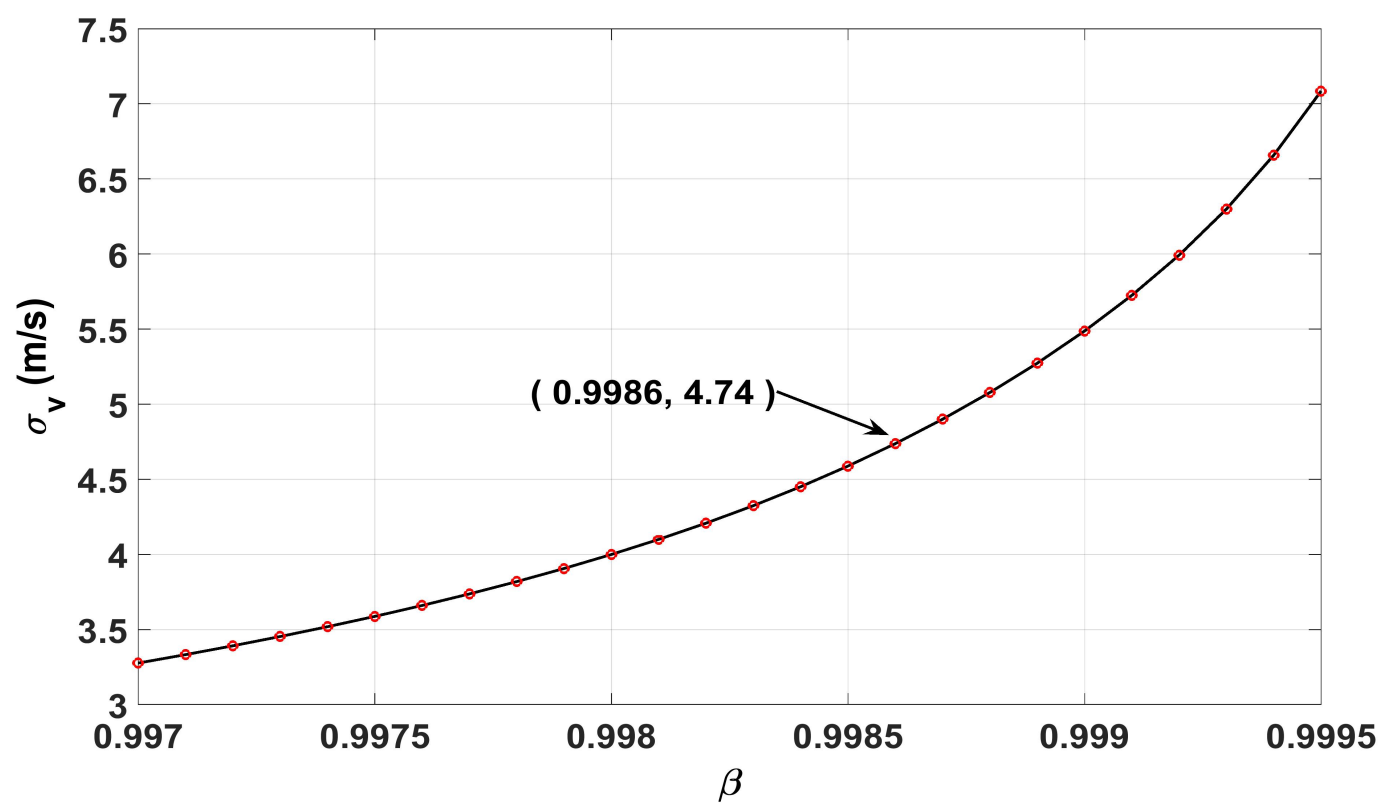

FIGURE 4.2: Velocity standard deviation vs the location of the high-pass filter pole

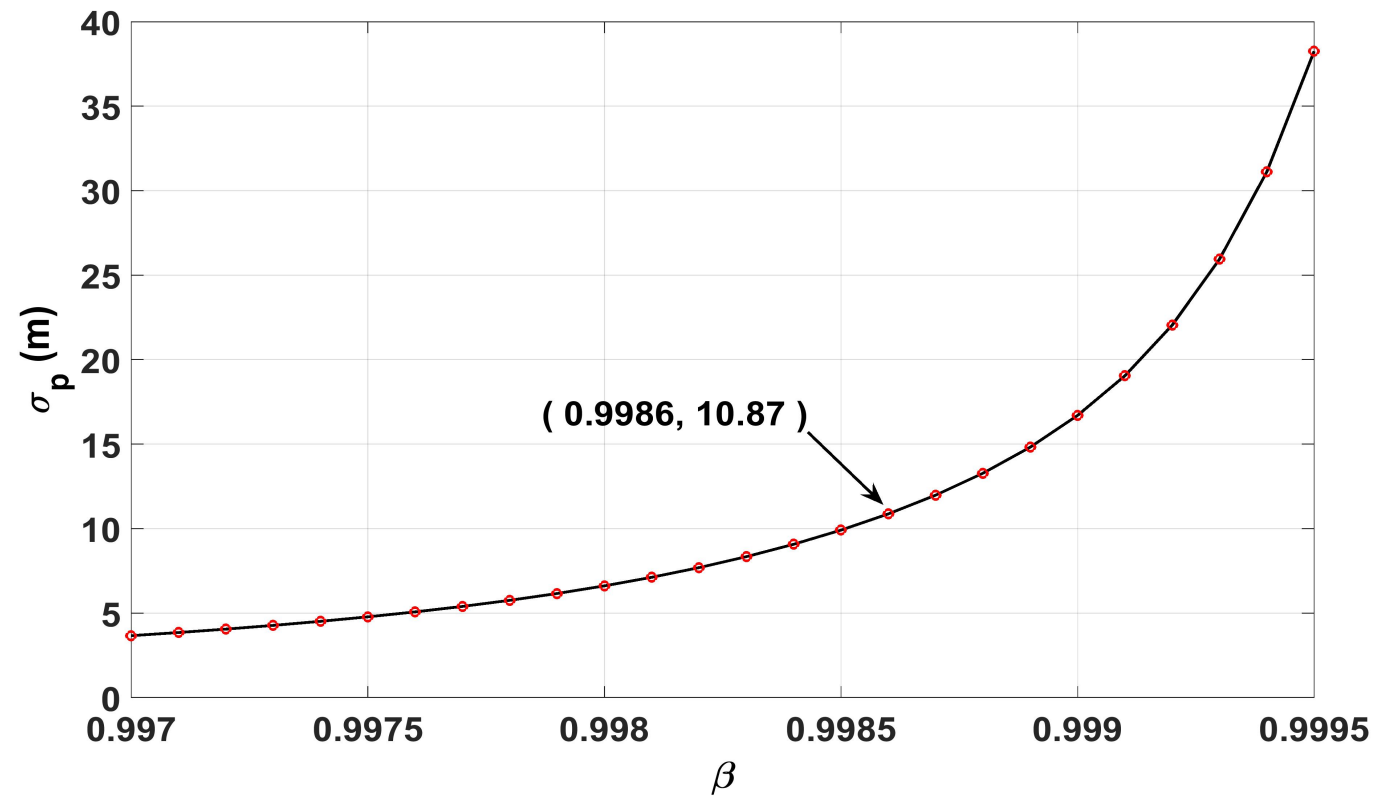

FIGURE 4.3: Position standard deviation vs the location of the high-pass filter pole 
The synthetic velocity and position were generated using an approximation of the recursive Newton-Euler equations of motion represented by 4.2 and 4.3 :

$$
\begin{aligned}
& \mathbf{v}_{n+1}=\frac{1}{f_{s}} \mathbf{a}_{n}+\mathbf{v}_{n} \\
& \mathbf{p}_{n+1}=\frac{1}{f_{s}} \mathbf{v}_{n}+\mathbf{p}_{n}
\end{aligned}
$$

Figure 4.4 demonstrates the synthetic acceleration, velocity and position.
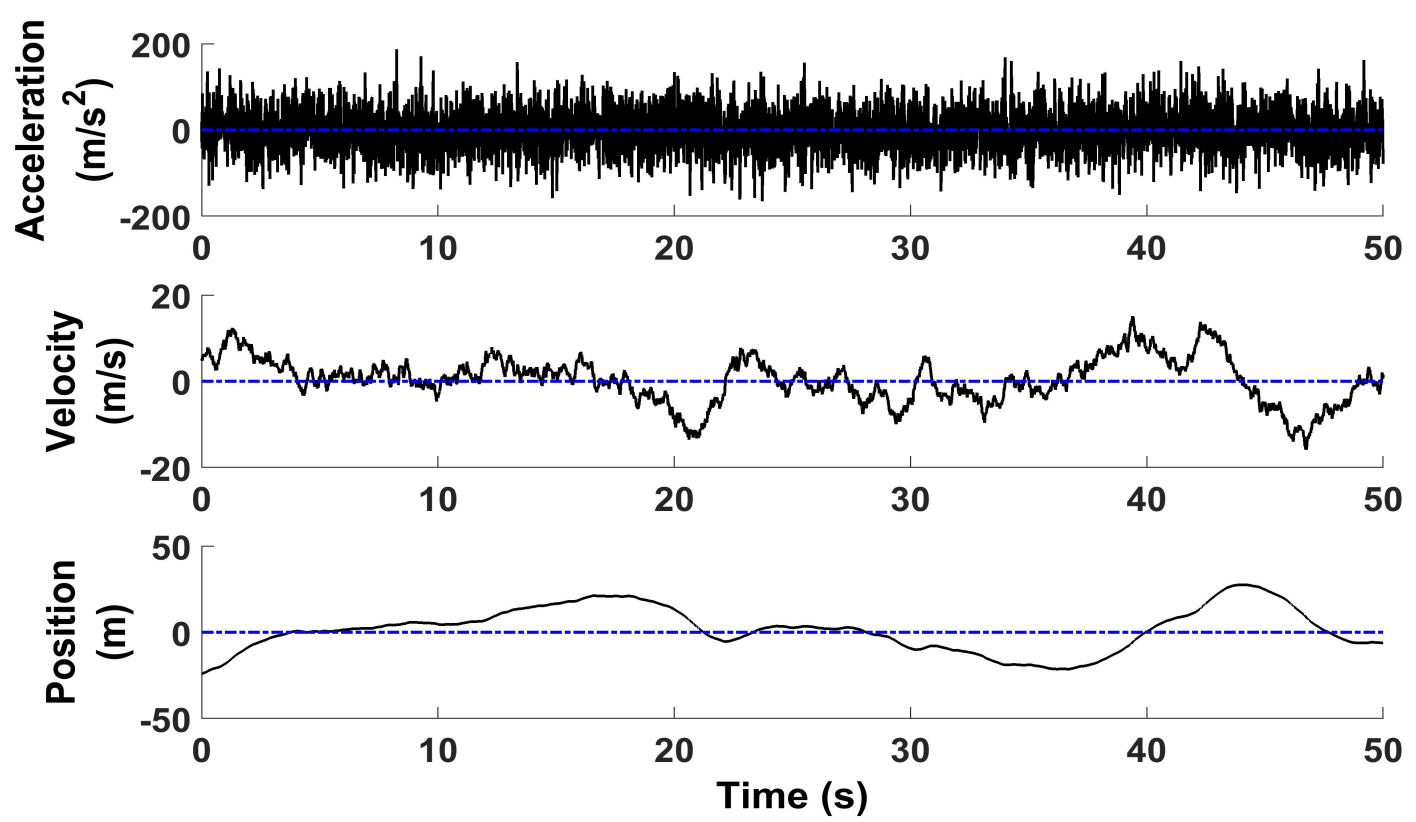

FIGURE 4.4: Top, middle and bottom panels demonstrate synthetic acceleration, velocity and position respectively.

3. Design of angular velocity: The angular velocity of the human motion, $\boldsymbol{\omega}_{n}$, is WSS signal whose low-frequency components have higher power than its high 
frequencies. Similar to the design process of the translational properties, the simplest approach to model $\boldsymbol{\omega}_{n}$ was to low-pass filter the WSS white noise sequence, $\mathbf{w}_{n}$. Figure 4.5 demonstrates the block diagram of the design of the angular velocity. The white noise represented the broadband angular acceleration, $\boldsymbol{\alpha}_{n}$. The low-pass filter was designed as a first order autoregressive model, $A R(1)$. Au-

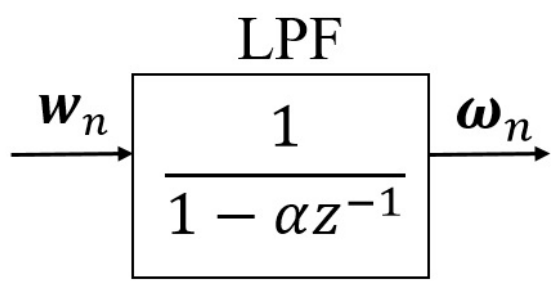

FIGURE 4.5: Block diagram of the design of the angular velocity, $\boldsymbol{\omega}_{n}$

toregressive models are stochastic processes in which the future values are estimated based on the weighted sum of the past values. In an $A R(1)$ process, the current value is calculated based on the immediate preceding value. Equation 4.4 represents the computation of the angular velocity:

$$
\boldsymbol{\omega}_{n+1}=\alpha \boldsymbol{\omega}_{n}+u_{n}
$$

where $u_{n}$ is a zero-mean white Gaussian noise. The value of the parameter $\alpha$ determines the behavior of an $A R(1)$ model; if $|\alpha|<1$, then the system will be bounded input, bounded output (BIBO) stable. There were two parameters to 
control the power of the high frequency components of the angular velocity and keep the standard deviation of the angular velocity within the range of 100 and $200^{\circ} / \mathrm{s}$ : the power of the white noise and the cutoff frequency of the low-pass filter. In order to have enough power for the high frequency components and fulfill the standard deviation constraint of the angular velocity, the value of $\alpha$ and the standard deviation of the white noise, $\sigma_{\mathbf{w}_{n}}$, were chosen as 0.7 and 1.8 respectively. As Figures 4.6 and 4.7 show the greater values led into generating higher $\sigma_{\boldsymbol{\omega}}$ and the smaller values resulted in reducing the standard deviation of the angular velocity.

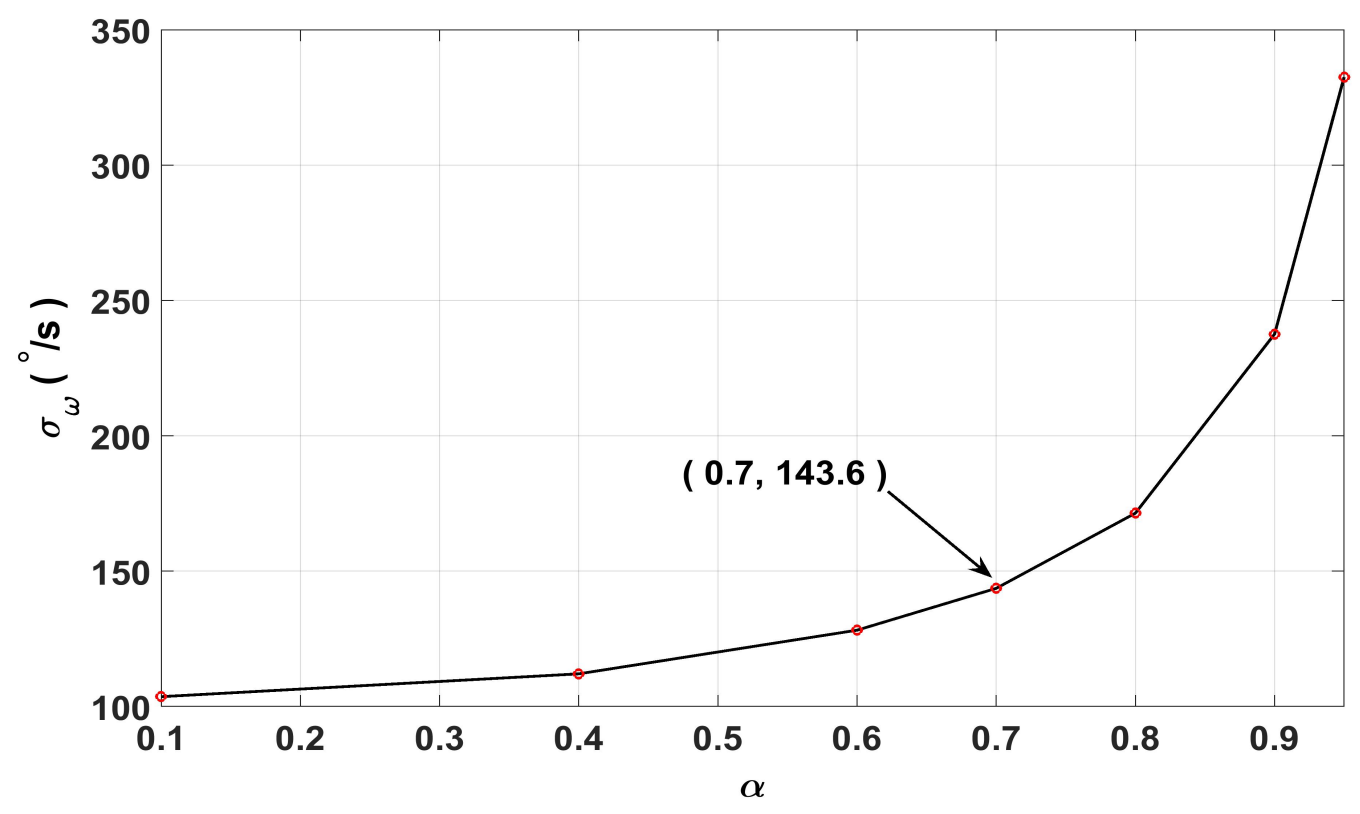

FIGURE 4.6: Angular velocity standard deviation vs $\alpha$ 


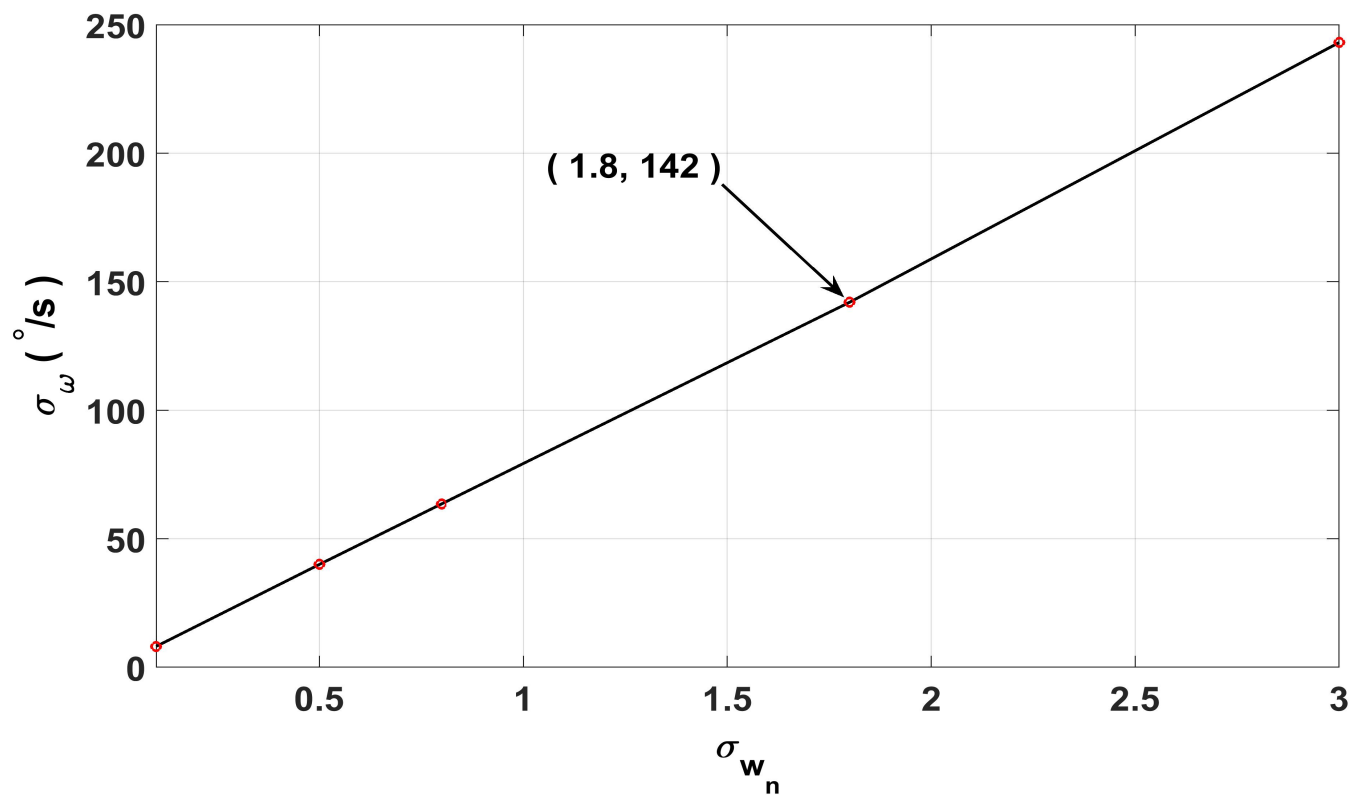

FIGURE 4.7: Angular velocity standard deviation vs White noise power

Figure 4.8 shows the synthetic angular velocity (top panel) and one of the synthetic Euler angles (bottom panel). 

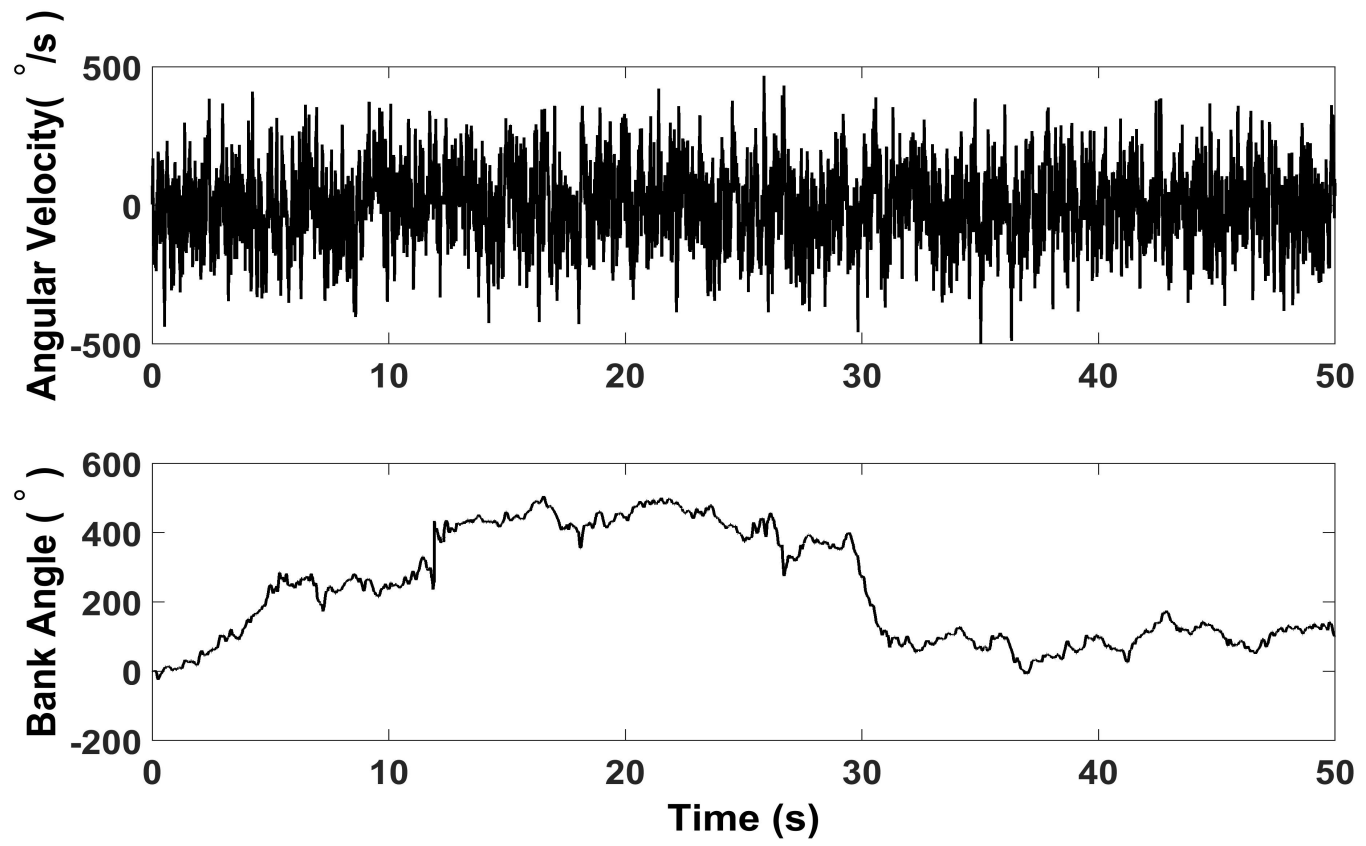

FIGURE 4.8: Top: Synthetic Angular Velocity, Bottom: Synthetic Bank Angle

\subsection{Experimental Design}

This section describes the evaluation of the performance of the short-term tracking algorithm using the IMU and an industrial robot arm as a reference system.

\subsubsection{Robot Arm}

Many of the previous studies have used optical systems as a way of assessing the human movements. However, as it was discussed in Chapter 1, optical systems suffer from many limitations such as high cost and extensive setup and calibration. Additionally, the application of the optical systems requires a laboratory environment where a clear line of sight between the source and the sensor is ensured. The industrial Epson 
C3 robot arm (Figure 4.9) is a 6-axis, high-speed robot used for a very high precision applications such as the lab automation, medical and industrial products and instrument panel assembly (Epson Robots, Carson, California). The robot has a wide range of motion and is accessible in the Portland State Biomedical Signal Processing Lab. Due to its high precision, wide motion range and accessibility, the robot arm was used as a reference measurement system to evaluate the performance of the inertial tracking algorithm.

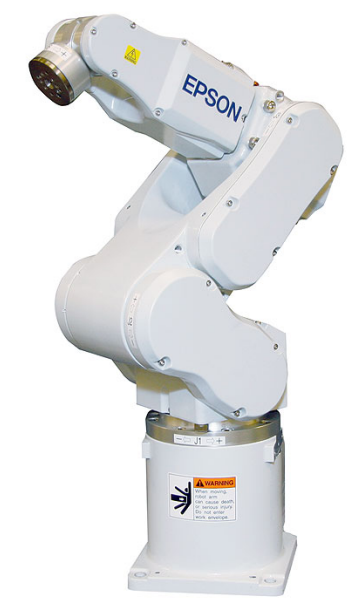

FIGURE 4.9: Epson C3 Robot Arm

The Opal sensor (Figure 4.10) is a miniature wireless IMU containing triaxial accelerometer and gyroscope. It has a long battery life of 16 hours and the ability of data storage up to 8 GB. Sensors are capable of synchronizing with other Opal sensors wirelessly, and the collected data has a precision of \pm 1 ms (APDM Inc., Portland, OR). The high performance specifications of Opal as well as its accessibility in the Portland State Biomedical Signal Processing Lab made Opal the best candidate for collecting data for this work. 


\begin{tabular}{|l||l|l|l|l|l|l|}
\hline \multicolumn{7}{|c|}{ Robot arm operating speed and motion range } \\
\hline Task Joint 1 & Joint 2 & Joint 3 & Joint 4 & Joint 5 & Joint 6 \\
\hline $\begin{array}{l}\text { Max operating } \\
\text { speed }\end{array}$ & $450^{\circ} / \mathrm{s}$ & $450^{\circ} / \mathrm{s}$ & $514^{\circ} / \mathrm{s}$ & $553^{\circ} / \mathrm{s}$ & $553^{\circ} / \mathrm{s}$ & $720^{\circ} / \mathrm{s}$ \\
\hline $\begin{array}{l}\text { Max motion } \\
\text { range }\end{array}$ & $\pm 180^{\circ}$ & {$[-160,65]^{\circ}$} & {$[-51,225]^{\circ}$} & $\pm 200^{\circ}$ & $\pm 135^{\circ}$ & $\pm 360^{\circ}$ \\
\hline $\begin{array}{l}\text { Resolution } \\
\left(\times 10^{-6}\right. \\
\text { pulse })\end{array}$ & 4.29 & 4.29 & 4.29 & 5.31 & 5.24 & 6.86 \\
\hline
\end{tabular}

TABLE 4.1: Epson Robots, Carson, California

The Opal gyroscope and accelerometer nominal noises are specified as spectral noise parameters corresponding to $0.025^{\circ} / \mathrm{s} / \sqrt{\mathrm{Hz}}$ and $120 \mu \mathrm{g} / \sqrt{\mathrm{Hz}}$ respectively (APDM, Inc., Portland, OR) which assumes that the noise is white with a uniform power spectral density for all the frequencies. The bandwidth of the anti-aliasing filter is $50 \mathrm{~Hz}$ for both the accelerometer and gyroscope. The root mean square (RMS) of the sensor noises is calculated through multiplying the noise spectral density by the square root of the noise bandwidth. Therefore, the RMS of the Opal accelerometer and gyroscope were measured as $0.0083 \mathrm{~m} / \mathrm{s}^{2}$ and $0.1768^{\circ} / \mathrm{s}$.

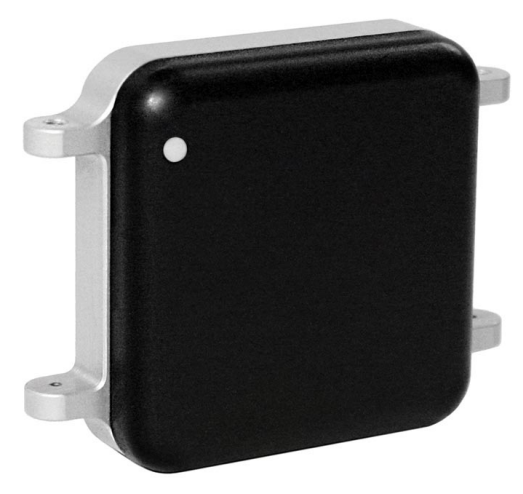

FIGURE 4.10: Opal Sensor

The movement protocol, originally designed for a different application, was found 
suitable for the application in this work to simulate the speed and complexity of the human movement. The Opal sensor was placed on the end effector of the robot arm with six degrees of freedom to measure the motion of the arm as demonstrated in Figure 4.11. In order to assess the performance of the short-term tracking algorithm for each of the three Euler angles and more easily find the deficiencies of the performance along each axis of rotation, the movement included approximately $20 \mathrm{~s}$ recording of the isolated motions about each of the three axes followed by a more complex motion consisting of simultaneous changes in the three Euler angles to resemble the human movement. The movement of the robot arm was programmed in four phases:

1. Sinusoidal movement only in the heading angle (about the Z-axis)

2. Sinusoidal movement only in the elevation angle (about $\mathrm{X}$-xis)

3. Sinusoidal movement only in the Bank angle (about Y-axis)

4. Simultaneous movements of all the three angles

Figure 4.12 shows the changes in the rotational angles of the robot arm, top panel shows the movement of the heading angle, while the changes in the elevation and bank angles are demonstrated in the middle and the bottom panels. 


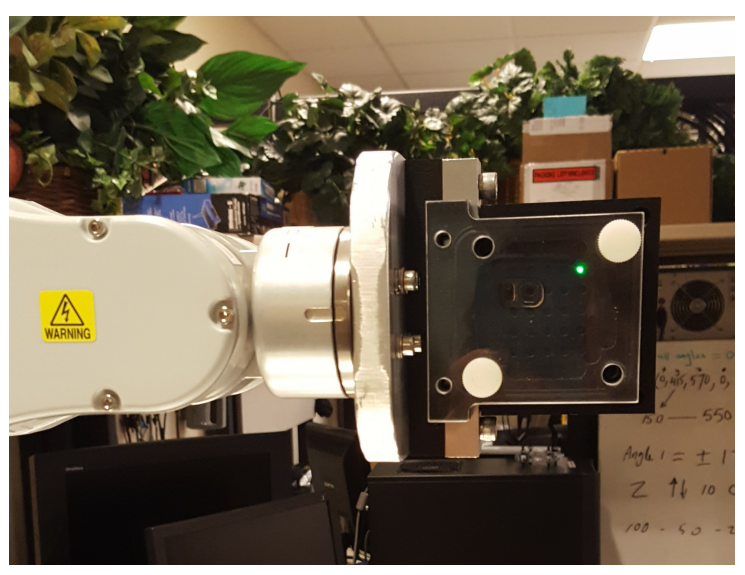

FIGURE 4.11: Robot End effector
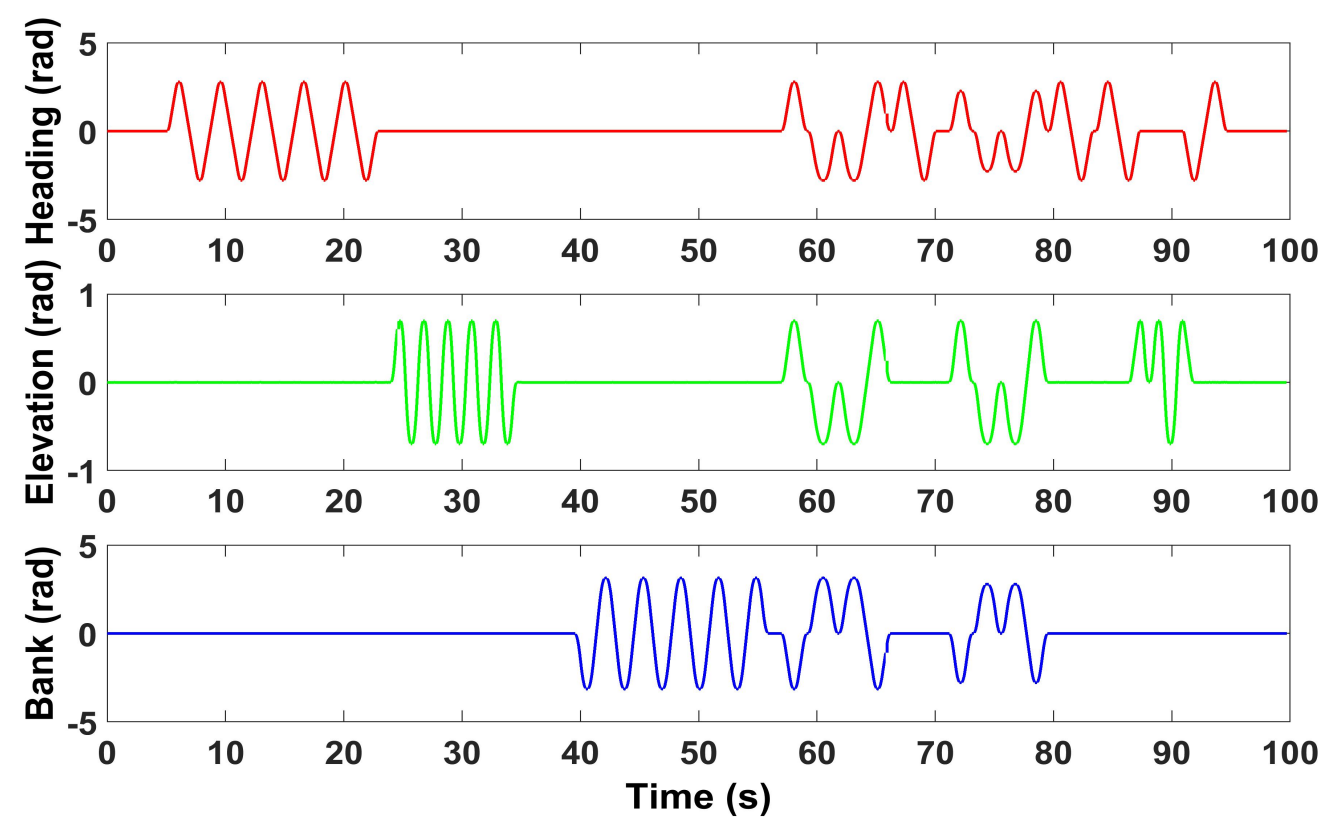

FIGURE 4.12: Robot Arm Orientation

\subsubsection{Data Preprocessing}

The movement of the robot arm was measured and recorded by the robot as well as the Opal sensor. The measurements taken by the robot arm were considered as the gold standard test to which the sensor measurements were compared. However, in 
order to compare the measurements, preprocessing the collected data was required. The preprocessing was done in the following steps:

- Alignment of the frames of reference

- Removing abrupt changes from the Euler angles measured by the robot arm

- Resampling the robot arm measurements

- Removing delay between the robot arm and the Opal measurements

\section{Frames of Reference Alignment}

Figure 4.13 demonstrates the coordinate systems of the base and the hand of the robot arm while Figure 4.14 shows the Opal frame of reference. In this thesis, the base of the robot was considered as the main reference frame and all the measurements were aligned with it. The alignment of the Opal sensor measurements were done by rotating the acceleration and the angular velocity from the sensor frame to the frame of reference of the robot arm's base.

\section{Removal of Abrupt Changes from the Robot Arm Euler Angles}

The next preprocessing job was done to remove the abrupt changes from the measured Euler angles by the robot arm. As discussed in Chapter 3, a unit quaternion can be presented in an angle-axis form, Equation 4.5.

$$
\mathbf{q}=\cos (\theta)+\mathbf{u} \sin (\theta)
$$

The negative quaternion $-\mathbf{q}$ is shown in Equation 4.6:

$$
-\mathbf{q}=\cos (2 \pi+\theta)+\mathbf{u} \sin (2 \pi+\theta)
$$




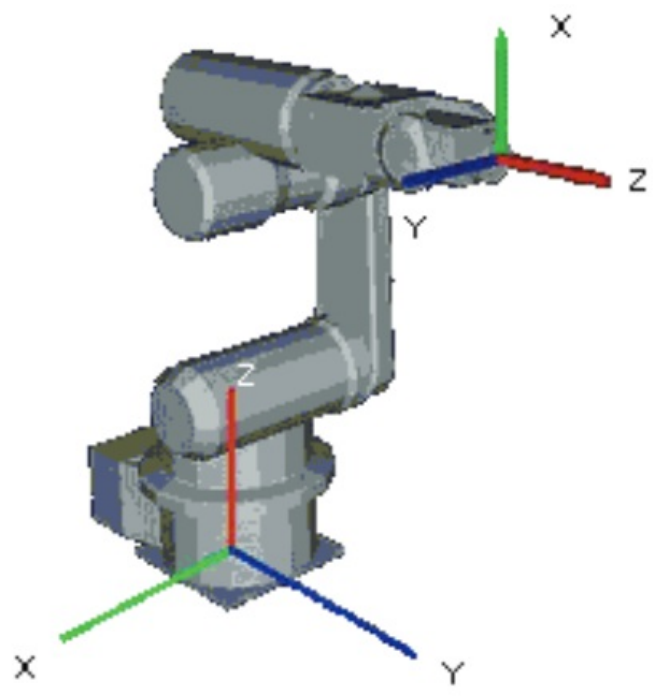

FIGURE 4.13: Robot Arm Coordinate System

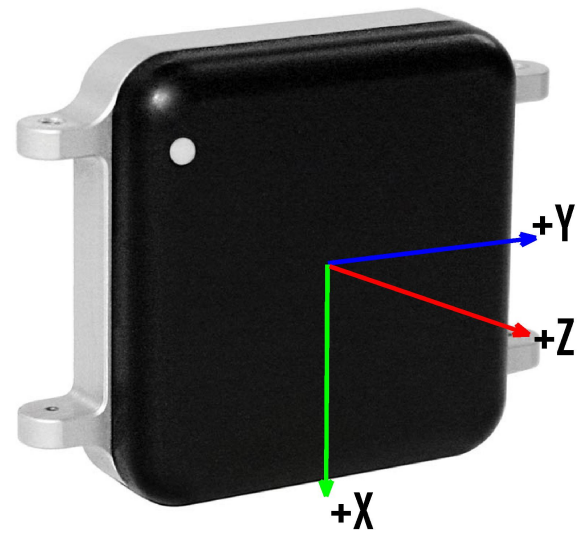

FIGURE 4.14: Opal Sensor Coordinate System

As Equations 4.5 and 4.6 demonstrate, the operations $L_{\mathbf{q}}$ and $L_{-\mathbf{q}}$ represent the same rotation about the same axis of rotation and through the same angle of rotation since $2 \pi+\theta$ is equivalent to $\theta$. 


$$
L_{-\mathbf{q}}=(-\mathbf{q}) \mathbf{v}(-\mathbf{q})^{*}=\mathbf{q} \mathbf{v}(\mathbf{q})^{*}
$$

The Euler angle signals collected by the robot arm were converted into quaternions in order to remove the gimbal lock. The gimbal lock refers to a situation when one rotational degree of freedom is lost because the two of the three rotation axes align. It usually happens when the second rotation in a sequence reaches 0 or $\pi / 2$ radians. The abrupt changes due to the redundancy in describing one rotation by two quaternions, $\mathbf{q}$ and $-\mathbf{q}$, were removed by finding the magnitude of the difference between $\mathbf{q}_{n}$ and $\mathbf{q}_{n-1}$ as well as $\mathbf{q}_{n}$ and $-\mathbf{q}_{n-1}$, and comparing the differences as described by equations 4.8-4.10:

$$
\begin{aligned}
& D^{+}=\left\|\mathbf{q}_{n}-\mathbf{q}_{n-1}\right\| \\
& D^{-}=\left\|\mathbf{q}_{n}-\left(-\mathbf{q}_{n-1}\right)\right\| \\
& \mathbf{q}_{n}= \begin{cases}-\mathbf{q}_{n}, & \text { if } D^{+}>D^{-} \\
\mathbf{q}_{n}, & \text { otherwise }\end{cases}
\end{aligned}
$$

\section{Resampling the Robot Arm Measurements}

The process of increasing the sampling frequency is called upsampling. The purpose of upsampling a signal, $s_{n}$, is to increase the sampling rate by a factor of $L$ which is equivalent to sampling the signal at every $T / L \mathrm{~s}$. In time domain processing, this can be interpreted as inserting $L-1$ zeros between the samples to form $s_{n / L}^{\prime}$. The DTFT of the upsampled signal is:

$$
S^{\prime}\left(e^{j \omega}\right)=\sum_{k=-\infty}^{\infty} x[k] e^{-j \omega L K}
$$


Due to having different sampling frequencies, it was required to change the sample rate of the Euler angles measured by the robot arm to the sample rate of the Opal sensor to obtain a higher bandwidth. The robot Euler angles were non-uniformly sampled at an average of $20 \mathrm{~Hz}$. The robot Euler angles were uniformly resampled at the sampling frequency of the Opal sensor, $128 \mathrm{~Hz}$. The Euler angles first were converted to a uniformly sampled signal by computing the average sample rate, and finding the rational sample ratio between the average sample rate and the desired sampling frequency and ultimately interpolating the signal. The cubic spline interpolation provides a smooth interpolated signal with higher accuracy than linear interpolation and avoiding the unwanted oscillations caused by the higher-order polynomial interpolation methods. Therefore the robot Euler angels were interpolated using the cubic spline method.

Figure 4.15 demonstrates the robot resampled Euler angles.
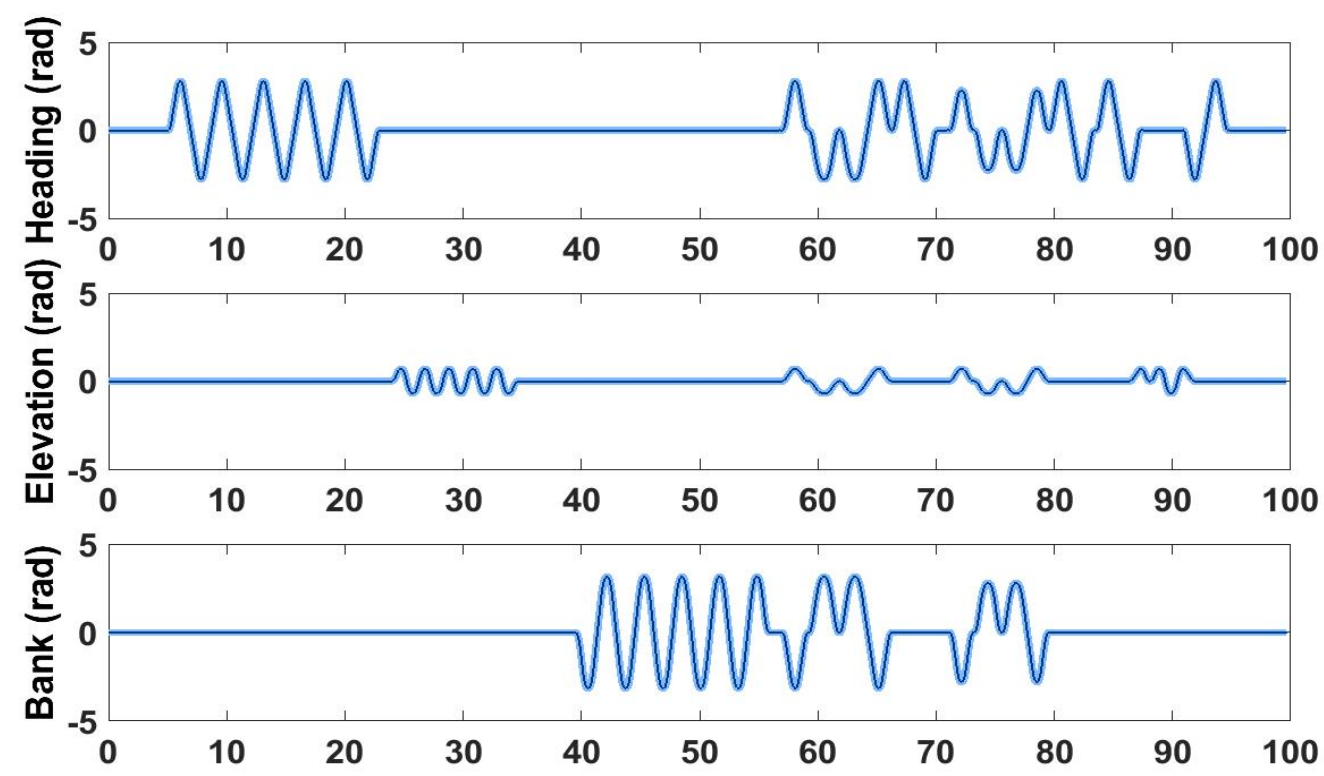

FIGURE 4.15: Overlap of the original robot Euler angles (light, thick blue) and resampled Euler angles (dark, thin blue) 


\section{Removal of Delay Between the Robot and Opal Measurements}

The measurements of the robot arm and the Opal sensor were recorded independently with different independent clocks which caused a delay between the measured Euler angles by the IMU and the robot Euler angles. The delay was calculated using crosscorrelation. The cross-correlation is a common technique for determining the time delay between two signals. The time delay is measured by calculating the maximum (or minimum if the signals are negatively correlated) of the cross-correlation signal (Rhudy et al., 2009). Equation 4.12 demonstrates the cross-correlation between the two stochastic processes, $y_{n}$ and $x_{n}$ :

$$
R_{y x}=\frac{\gamma_{y x}(l)}{\sigma_{y} \sigma_{x}}
$$

where $l$ is the lag, $\gamma_{y x}$ is the cross-covariance of $y_{n}$ and $x_{n}$ and $\sigma_{y}$ represents the standard deviation of the corresponding process.

Figure 4.16 indicates the maximum lags between the three channels of the robot and Opal measurements equivalent to the time delay which was measured as $13.02 \mathrm{~s}$. 


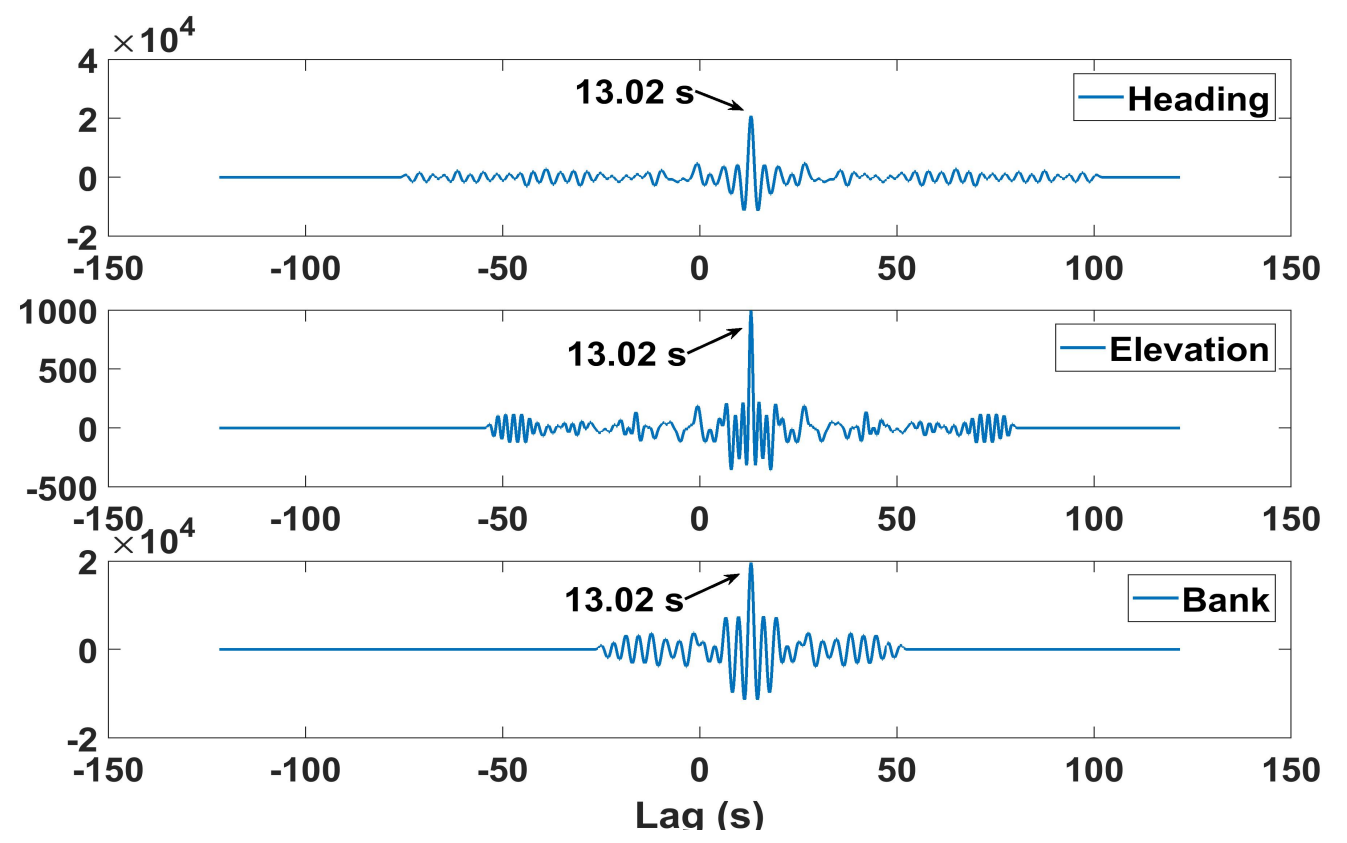

FIGURE 4.16: Cross-correlation of Opal and robot Euler angles

\subsection{Euler Angles Performance Assessment}

The proposed algorithm only utilizes the accelerometer and gyroscope measurements to estimate the orientation. The tracker uses gravity to correct for the estimation of the elevation and bank angles. Previous studies have employed magnetometers to find the magnetic field of earth and compensate for the heading errors. As it was carefully discussed in Chapter 2, the presence of magnetic disturbances greatly affects the accuracy of the estimation of the heading angle. Due to not using the magnetometer in this work, there was no absolute reference for measuring the rotation about the vertical axis, equivalently the heading angle. Therefore, this work is only concerned about the assessment of the estimation of the elevation and bank angles.

There are at least two unit quaternions that represent every orientation. If $\mathbf{q}$ is one representation, then $-\mathbf{q}$ is another. In order to compare the estimated and true rotational angles, it was easier to convert the estimated quaternions into Euler angles since 
the quaternions do not segregate the heading error from the error in the elevation and bank angles. On the other hand, Euler angles suffer from singularity, which is also known as gimbal lock problem. In order to prevent the gimbal lock issue in this work, different Euler angle-axis sequences (explained in detail in Chapter 3) were examined and the one which avoided the singularity problem was utilized for the conversion of the quaternions to Euler angles. Furthermore, in Euclidean geometry the sum of the even integer multiples of $\pi$ and an angle is equivalent to the angle itself $(\gamma$ and $\gamma+2 n \pi$ are equivalent), in practice this can result in the discontinuities and jumps of $\pm 2 \pi$ when working with the Euler angles. In order to keep the values of the Euler angles between $-\pi$ and $\pi$ and prevent the discontinuities, the estimated Euler angles were unwrapped. The process of unwrapping was done by adding appropriate multiples of $2 \pi$ to each phase input to find the unambiguous phase values.

The median absolute deviation (MAD) is a robust scale estimator which is significantly less influenced by the outliers compared to the conventional estimators such as root mean square deviation (RMSD) or mean absolute error. The MAD measures the absolute difference between each sample and the median of a dataset. In order to assess the accuracy of the estimated elevation and bank angles, the MAD between the estimated and true Euler angles was calculated for different future feedback durations, $N$, Equation 4.13:

$$
\operatorname{MAD}_{N}=\underset{N}{\operatorname{median}}\left(\left|\theta_{n}-\operatorname{mean}_{i}\left(\hat{\theta}_{n}^{i}\right)\right|\right)
$$

where $\hat{\theta}_{n}^{i}$ represents the estimated angle for the $i$-th realization. 


\section{Results and Discussion}

This chapter provides the results of the short-term tracking algorithm performance for the synthetic and real data. Section 5.1 presents the performance of the proposed method for different number of seconds in the future for the synthetic data. Furthermore, it compares the performance of the short-term tracking algorithm with the results obtained from the inertial navigation approach. Section 5.2 describes the similar algorithm performance for the real data.

\subsection{Algorithm Performance Using Synthetic Data}

Figure 5.1 shows the median absolute deviation (MAD) between the estimated and true elevation and bank angles for a synthetic dataset with a duration of $2000 \mathrm{~s}$. Due to the presence of the gimbal lock in the absolute differences of the true and estimated angles, the MAD was chosen as a robust method for measuring the variability of the error. The blue line represents the MAD between the true Euler angles and the ones obtained from the inertial navigation approach for the average of 50 realizations. The inertial navigation technique is a self-contained navigation system which does not depend on external infrastructure. It uses the accelerometer and gyroscope measurements to track the position and orientation relative to a known initial position, orientation and velocity. The red line in Figure 5.1 indicates the MAD between the true and estimated angles measured by the short-term tracking algorithm for $2.5 \mathrm{~s}$ in the future and over the average of 10 realizations. It is clearly evident that applying the short-term tracking algorithm significantly decreases the amount of orientation estimation error. The blue 
trace is smoother than the red one since it is obtained from the average of larger number of realizations; the amount of time required to estimate the orientation using the shortterm tracking algorithm was so high that limited the feasibility of estimation for greater number of realizations.
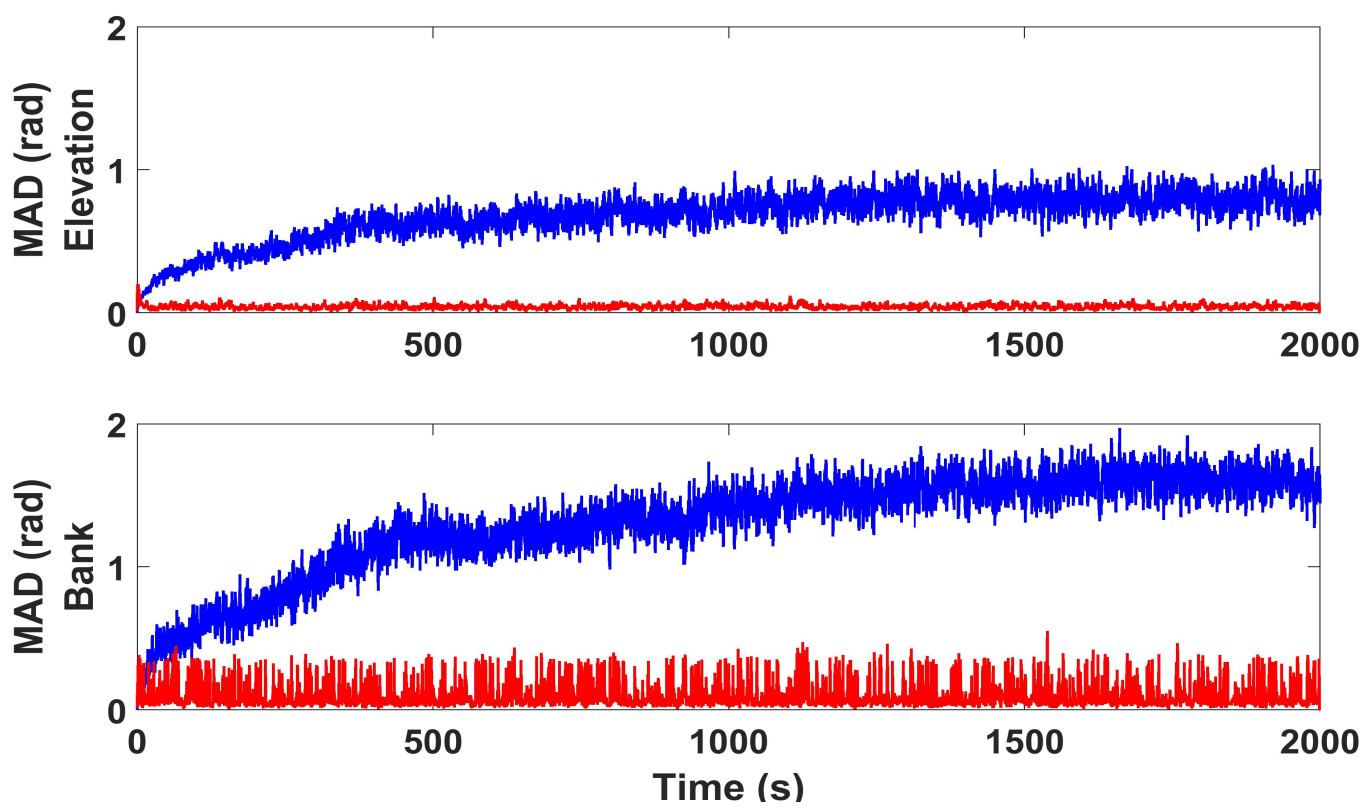

FIGURE 5.1: Median absolute error between the true and estimated Euler angles: the blue line represents the MAD between the true and estimated angles by inertial navigation method for 50 realizations, the red line shows the MAD between the true and estimated angles measured by the new technique for 10 realizations.

Due to initializing the tracking algorithm with the true initial state, the absolute error between the true and estimated sequences consists of a transient and steady state. Figure 5.2 shows the MAD of the steady state for 0 to $2.5 \mathrm{~s}$ in the future. The dotted horizontal lines indicate the MAD of the elevation and bank angles using the inertial navigation approach as the reference point for assessing the performance of the shortterm tracking algorithm. 
Figure 5.2 clearly shows that the proposed method significantly improves the estimation of the elevation and bank angles compared to applying the inertial navigation only. Table 5.1 lists the MAD of the elevation and bank angles demonstrated in Figure 5.2. The error for both angles continues to decrease as going further in the future.
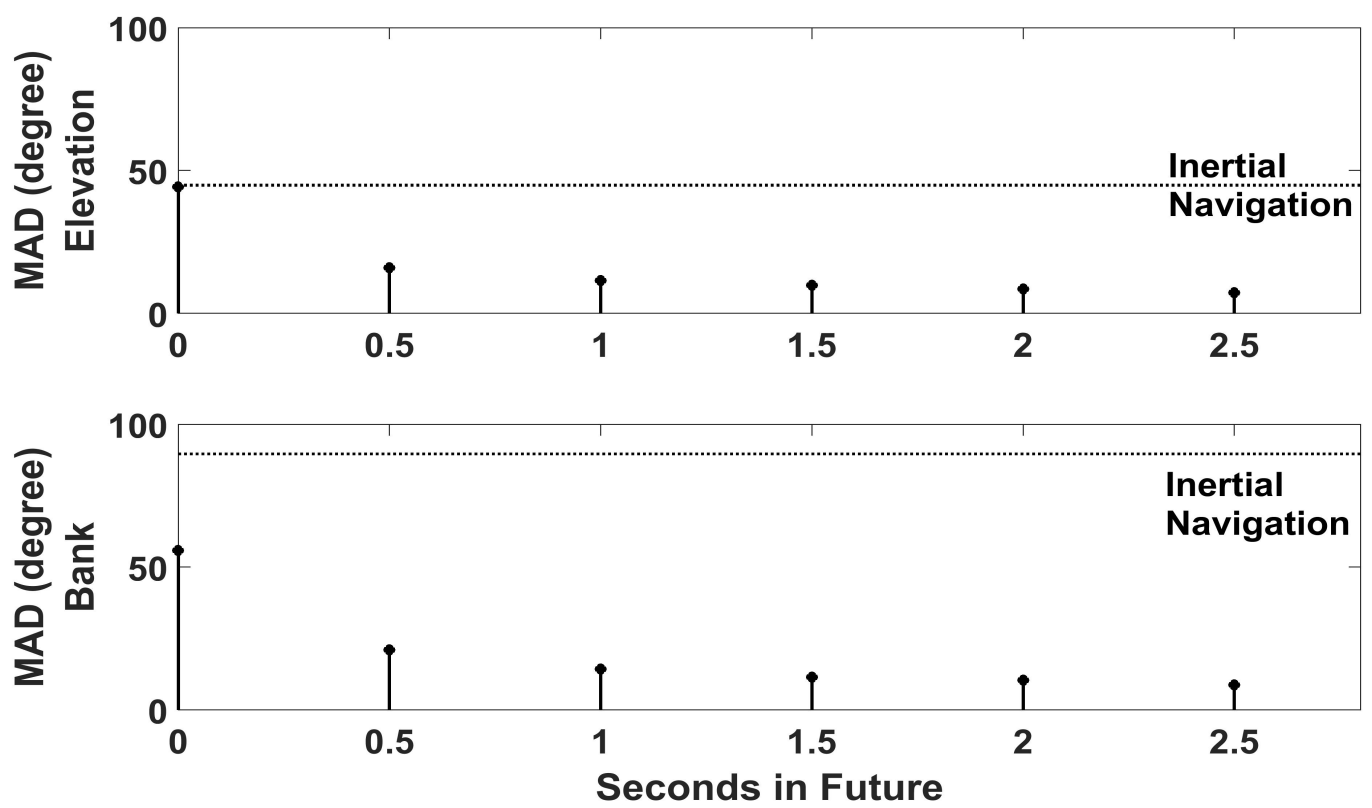

FIGURE 5.2: Comparison of performance of inertial navigation approach with the short-term tracking algorithm for different number of seconds in the future on the synthetic data.

\begin{tabular}{|l||l|l|l|l|l|l|l|}
\hline \multicolumn{8}{|c|}{ Median Absolute Deviation of Euler Angles } \\
\hline Parameter Name & $\begin{array}{l}\text { Inertial } \\
\text { navigation }\end{array}$ & $0 \mathrm{~s}$ & $0.5 \mathrm{~s}$ & $1 \mathrm{~s}$ & $1.5 \mathrm{~s}$ & $2 \mathrm{~s}$ & $2.5 \mathrm{~s}$ \\
\hline $\begin{array}{l}\text { MAD of Eleva- } \\
\text { tion angle }\left(^{\circ}\right)\end{array}$ & 44.9 & 44.2 & 16.0 & 11.5 & 9.79 & 8.53 & $\mathbf{7 . 2 0}$ \\
$\begin{array}{l}\text { MAD of Bank an- } \\
\text { gle }\left({ }^{\circ}\right)\end{array}$ & 89.6 & 55.8 & 21.0 & 14.3 & 11.3 & 10.5 & $\mathbf{8 . 8 0}$ \\
\hline
\end{tabular}

TABLE 5.1: Median absolute deviation of the elevation and bank angles for synthetic data 


\subsection{Algorithm Performance Using Real Data}

The short-term tracking algorithm was further evaluated by applying it to real data collected by an actual IMU and a robot arm. The original recorded real data was replicated in time to obtain a longer dataset. Figures 5.6 and 5.7 illustrate the overlay of the true and estimated elevation (top panel) and bank (bottom panel) angles. The thicker, black lines show the true angles while the thinner lines illustrate the estimated angles using the short-term tracking algorithm (Figure 5.6) and inertial navigation approach (Figure 5.7).
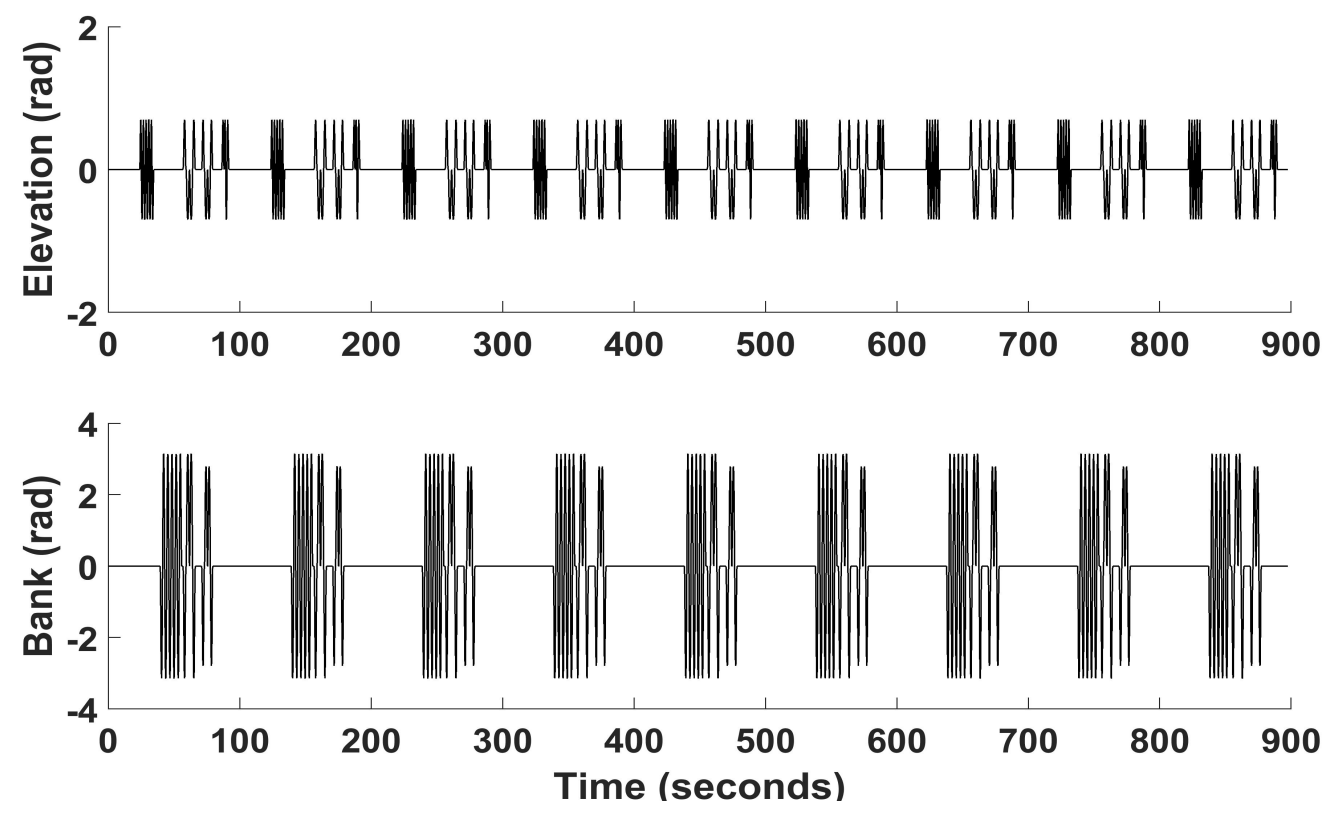

FIGURE 5.3: True elevation (top panel) and bank (bottom panel) angles 

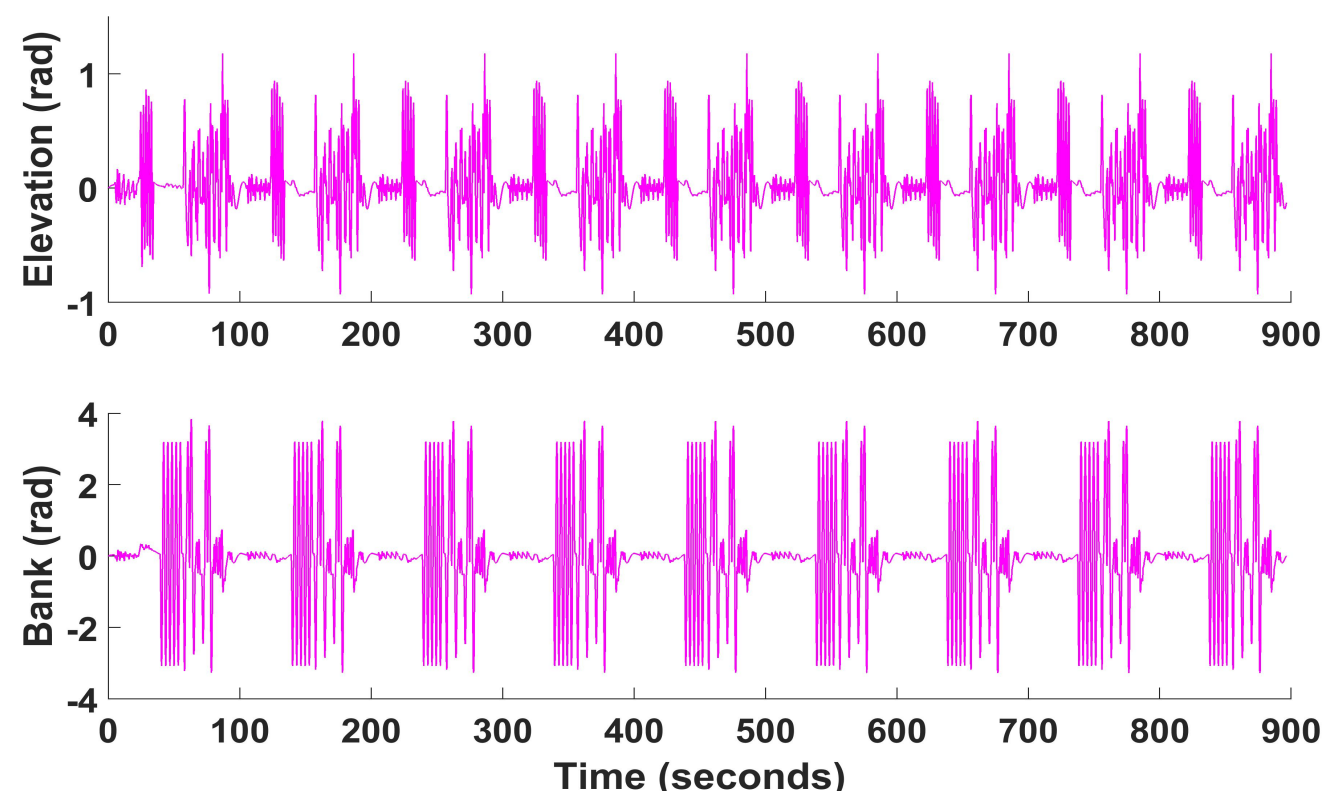

FIGURE 5.4: Estimated elevation (top panel) and bank (bottom panel) angles by short-term tracking algorithm
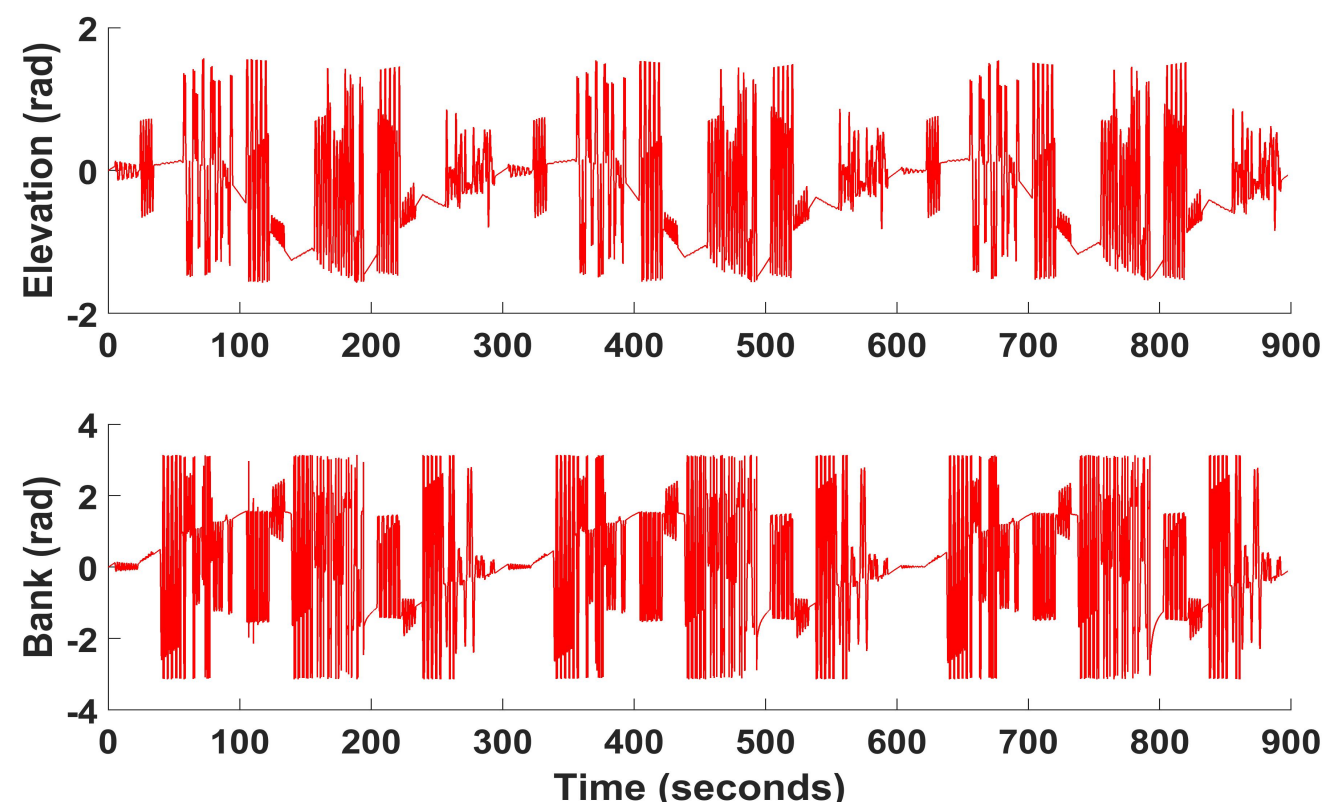

FIGURE 5.5: Estimated elevation (top panel) and bank (bottom panel) angles by inertial navigation approach 

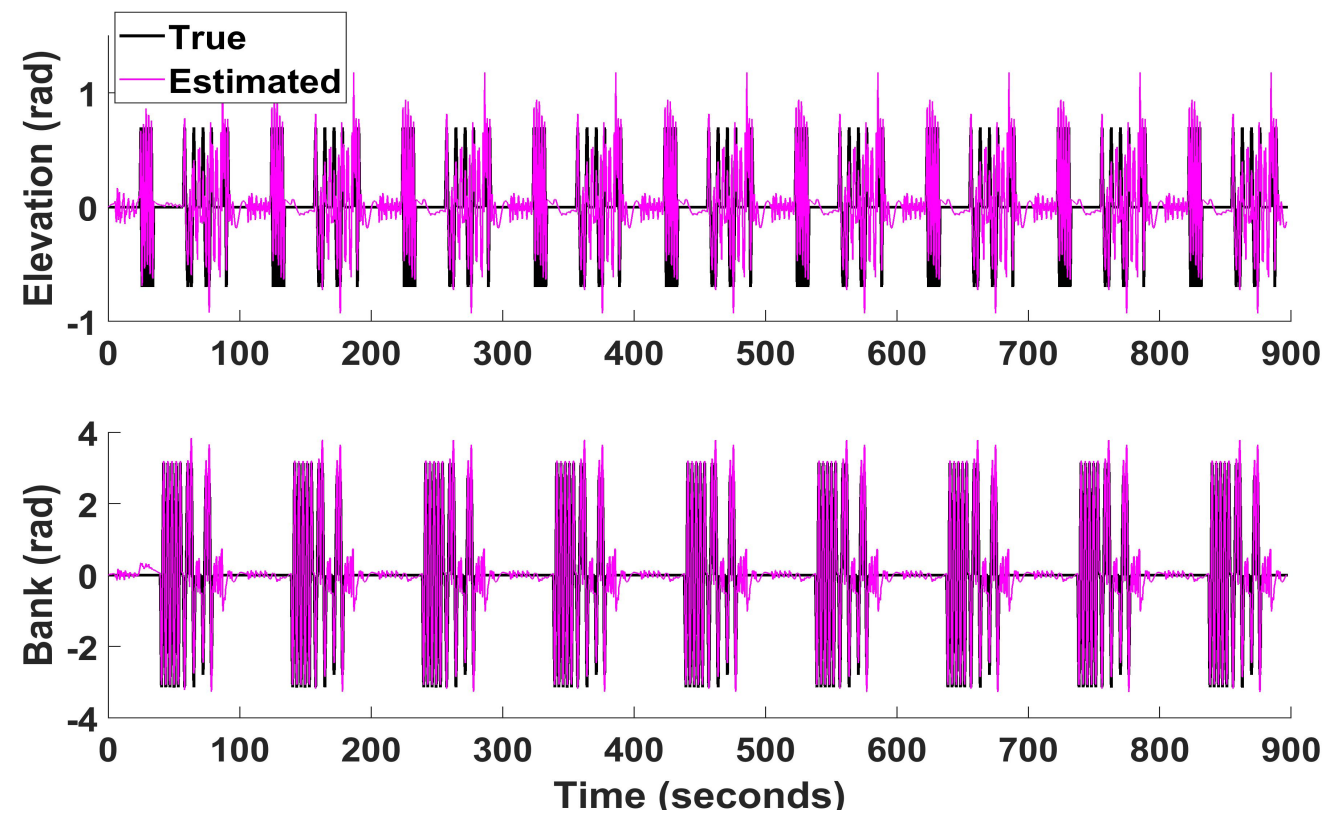

FIGURE 5.6: The overlay of the true and estimated elevation (top panel) and bank (bottom panel) angles. The black lines show the true angles and the magnum lines illustrate the estimated angles using the short-term tracking algorithm. 

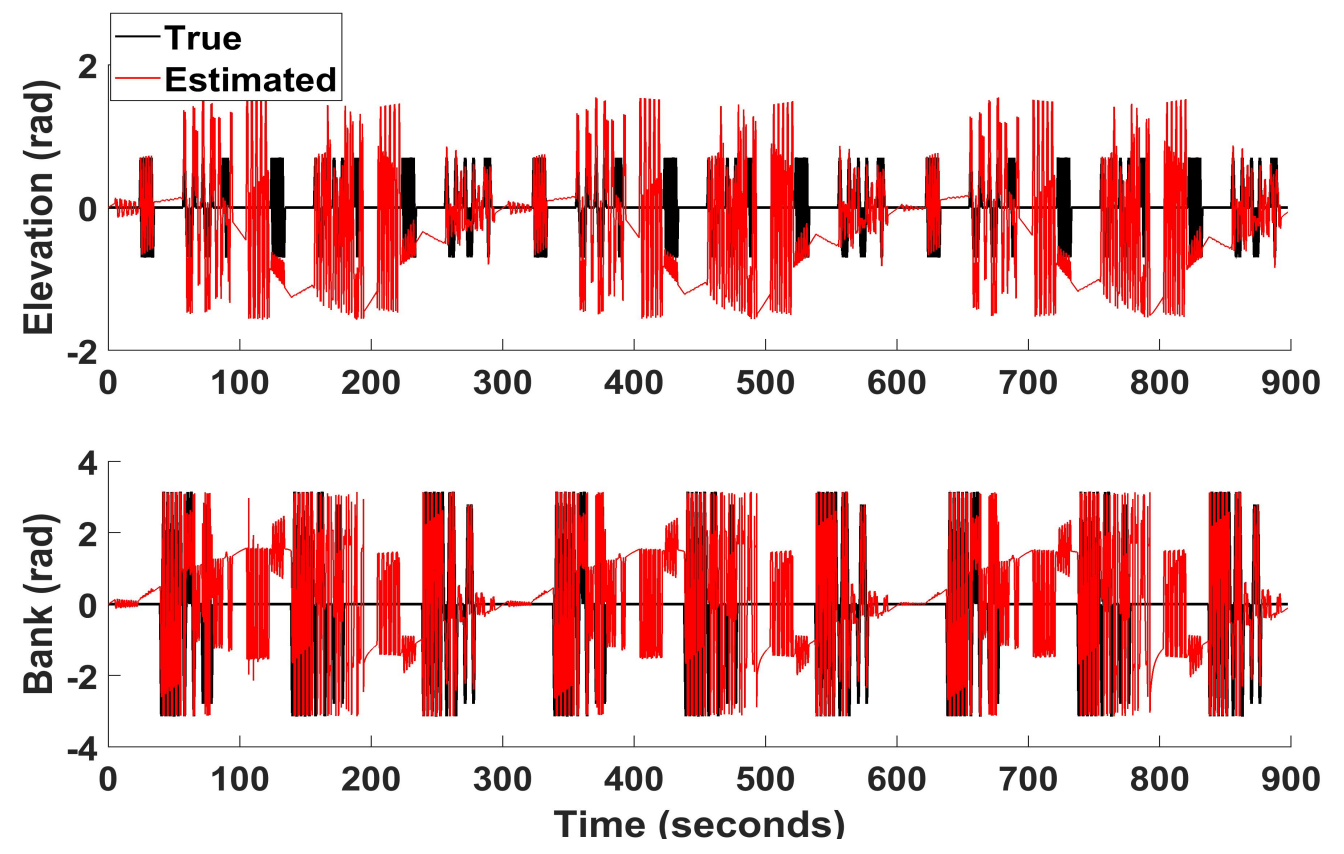

FIGURE 5.7: The overlay of the true and estimated elevation (top panel) and bank (bottom panel) angles. The black lines show the true angles and the red lines illustrate the estimated angles using the inertial navigation approach.

The MAD was calculated between the estimated and true elevation and bank angles for a recording with the duration of 900 s. Figure 5.8 shows the MAD of the short-term tracking algorithm for 0 to $2 \mathrm{~s}$ in the future. The dotted horizontal lines obtained from the inertial navigation approach serve as the point of reference for assessing the shortterm tracking algorithm performance. Table 5.2 lists the MAD of the elevation and bank angles. The results indicate that applying the short-term tracking algorithm considerably decreases the estimation error. The error continues to decrease as the number of seconds in the future increases. 

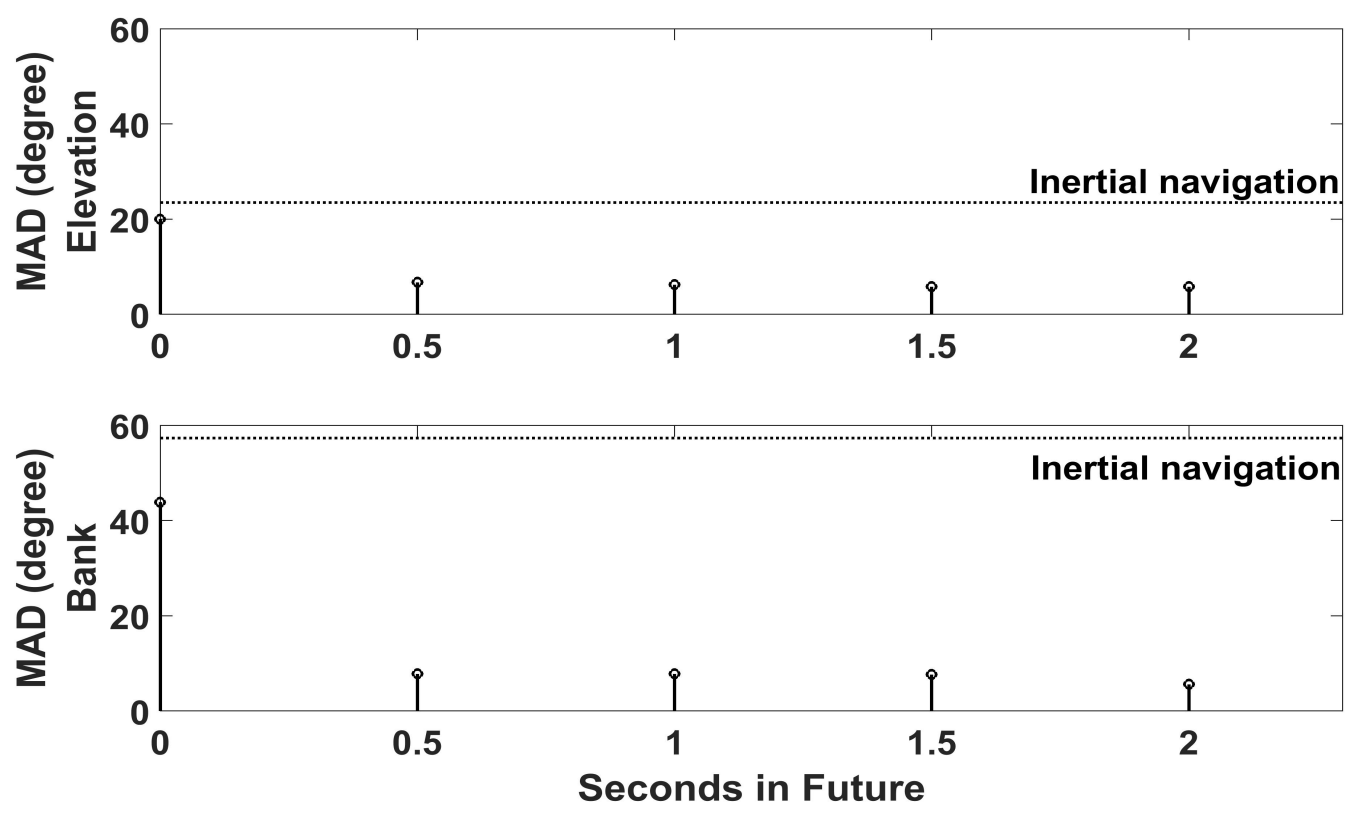

FIGURE 5.8: Comparison of performance of inertial navigation approach with the short-term tracking algorithm for different number of seconds in the future on the real data.

\begin{tabular}{|l||l|l|l|l|l|l|}
\hline \multicolumn{7}{|c|}{ Median Absolute Deviation of Euler Angles } \\
\hline Parameter Name & $\begin{array}{l}\text { Inertial } \\
\text { navigation }\end{array}$ & $0 \mathrm{~s}$ & $0.5 \mathrm{~s}$ & $1 \mathrm{~s}$ & $1.5 \mathrm{~s}$ & $2 \mathrm{~s}$ \\
\hline $\begin{array}{l}\text { MAD of Eleva- } \\
\text { tion angle }\left(^{\circ}\right) \\
\begin{array}{l}\text { MAD of Bank an- } \\
\text { gle }\left({ }^{\circ}\right)\end{array}\end{array}$ & 23.5 & 20.0 & 6.77 & 6.24 & 5.84 & 5.83 \\
\hline
\end{tabular}

TABLE 5.2: Median absolute deviation of the elevation and bank angles for the real data 


\section{Conclusion}

The first section of this chapter, 6.1 provides a summary of the thesis. Section 6.2 describes the contribution of the thesis to the area of motion capturing. Section 6.3 enumerates the limitations of the current work and potential future research. Finally, Section 6.4 provides a conclusion of the thesis.

\subsection{Summary}

Recently, the application of the IMU's has been increased for continuous measurement of the orientation of a moving body. While inertial navigation is a typical approach to estimate the orientation, this approach has a major problem which is the accumulation of error in orientation estimation when integrating the sensor measurements. Chapter 2 of this thesis reviewed and summarized the current solutions to address this problem. One of the constraints of the current practices includes limited tracking duration, up to $20 \mathrm{~s}$, for a controlled movement. Most of the present practices apply zero velocity update as a method of calibration to improve the accuracy of the orientation estimation. The main limitation of this approach is that it requires the sensors to be stationary frequently. Application of the magnetometer is the other common practice to improve the orientation estimation about the vertical axis (heading angle), but the accuracy of this method significantly suffers from the sensitivity of the magnetometer to the environmental magnetic disturbances.

This thesis proposed a more accurate algorithm to estimate the elevation and bank 
angles of the orientation of the human movement over a persistent motion using accelerometers and gyroscopes. The introduced method incorporates the knowledge about the human movement to the estimation of the orientation to restrict the estimated position from growing unboundedly. As it was discussed in details in Chapter 3, the proposed measurement model utilizes the accelerometer and gyroscope measurements to estimate the position $T$ seconds in the future. The estimated position is used to help with increasing the reliability of using the measurement model in the UKF without the need for the accurate reading of gravity by the accelerometer which requires the sensor to be stationary. Chapter 4 described designing a model to simulate the human movement and presented a method to thoroughly assess the performance of the algorithm on the synthetic data. In addition, Chapter 4 explained an experimental design where the real data was captured using an IMU and a robot arm, and was preprocessed before being used by the short-term tracking algorithm. Ultimately, Chapter 5 presented the performance results of the new method and compared it with the inertial navigation performance.

\subsection{Contribution}

This thesis contributes to the area of motion tracking using IMU's by introducing a novel method of calibrating the orientation estimation. The four major contributions from this work are:

- introducing a new method for estimating orientation by incorporating the prior knowledge about human movement into the tracking algorithm,

- designing a model for the human motion,

- rigorously assessing the introduced method on the synthetic data, and 
- evaluating the performance of the proposed tracker on the real data captured by an actual IMU.

\subsection{Limitations and Future Research}

There are few limitations associated with the short-term tracking algorithm. These limitations include:

- The main limitation of the short-term tracking algorithm is that it is computationally expensive. The tracker takes a significant amount of time to do the estimation for long recordings. The algorithm is designed to compute the estimated position for every single sample in the future; therefore, as the sampling frequency of the dataset or the duration of the recording increases, the time required for estimating the orientation grows significantly. The current algorithm requires more future work to optimize the computational efficiency of the tracker while maintaining an acceptable level of estimation accuracy.

- It is important to assess the short-term tracking algorithm on different types of datasets which include a variety of movement captured by the robot arm as well as motion capture settings.

- Future work needs to be done to investigate the number of seconds in the future which would provide the best estimation performance for various datasets, and determine whether this number is independent of the type of the movement or it is a function of the complexity of the motion.

- It is feasible to estimate the position using the short-term tracking algorithm. As previous chapters discussed, the position signal of the human movement has higher power for the lower frequency components. Estimation of the coherence 
between the estimated and true position can be a potential approach to evaluate the accuracy of the position estimation.

- In 2003, Kraft described a quaternion-based UKF for orientation tracking which is an extension to the original one. The proposed extension addresses the inherent properties of the unit quaternions (Kraft, 2003). Future work needs to be done to utilize the proposed quaternion-based UKF in the short-term tracking algorithm for estimating the orientation and avoiding some of the intrinsic problems of the unit quaternions.

- This work only used the short-term tracking algorithm and the measurements from the accelerometer and gyroscope to estimate the elevation and bank angles of human motion. Future work needs to be done to integrate the ZUPT in to the new technique as well as utilize magnetometers to improve the heading angle estimation. It is valuable to determine how much the short-term tracking algorithm improves the estimation accuracy in addition to the ZUPT during a typical human movement.

\subsection{Conclusion}

This thesis demonstrated the potential of estimating the orientation of the human motion more accurately over a persistent motion using the inertial measurement units by incorporating the prior knowledge about the movement into the tracking algorithm. The results presented in this work confirmed that the introduced method considerably improves the accuracy of the orientation estimation compared to the inertial navigation approach. A model of the human movement was designed and the performance of the algorithm was evaluated on the synthetic data. Additionally, the real data was collected using an actual IMU and a robot arm for further assessment of the proposed technique. 
The results for both datasets indicated that the estimation of the elevation and bank angles significantly were improved using the short-term tracking algorithm. Further future work is required to assess the performance of the algorithm for a wider variety of motion collected by the robot arm as well as motion capture settings. Furthermore, it is important to investigate the amount of orientation estimation improvement after integrating the ZUPT in to the short-term tracking algorithm and evaluating the accuracy of the estimation for the human movement. 


\section{Bibliography}

Abdulrahim, K. et al. (2014). "Rotating A Mems Inertial Measurement Unit For A Foot-Mounted Pedestrian Navigation". In: Journal of Computer Science 10.12, pp. 2619-2627.

Alvarez, J. C. et al. (2012). "Pedestrian dead reckoning with waist-worn inertial sensors". In: 2012 IEEE International Instrumentation and Measurement Technology Conference Proceedings, pp. 24-27.

Arulampalam, M. S. et al. (2002). “A tutorial on particle filters for online nonlinear / non-Gaussian Bayesian tracking". In: IEEE Transactions on Signal Processing 50.2, pp. 174-188.

Bachmann, E. R. (2000). “Inertial and Magnetic Tracking of Limb Segment Orientation for Inserting Humans in Synthetic Environment". In: PhD thesis, Naval Postgraduate School.

Bachmann, E. R., Xiaoping Yun, and C. W. Peterson (2004). “An investigation of the effects of magnetic variations on inertial/magnetic orientation sensors". In: Robotics and Automation, 2004. Proceedings. ICRA '04. 2004 IEEE International Conference 2, pp. 1115-1122.

Barbour, N. and G. Schmidt (2001). "Inertial sensor technology trends". In: IEEE Sensors Journal 1.4, pp. 332-339. 
Bergmann, R. Mayagoitia, and I. Smith (2009). "A portable system for collecting anatomical joint angles during stair ascent: a comparison with an optical tracking device". In: Dynamic Medicine 8.3, pp. 1-7.

Dam, A. van et al. (2000). "Immersive VR for scientific visualization: a progress report". In: IEEE Computer Graphics and Applications 20.6, pp. 26-52.

Dam, Erik B, Martin Koch, and Martin Lillholm (1998). Quaternions, interpolation and animation. Vol. 2.

El-Gohary, Mahmoud Ahmed (2013). “Joint Angle Tracking with Inertial Sensors". In: Dissertations and Theses, p. 661.

Foxlin, E. (1996). “Inertial head-tracker sensor fusion by a complementary separate bias Kalman filter". In: Proceedings of the IEEE 1996 Virtual Reality Annual International Symposium, Santa Clara, CA, pp. 185-194,267.

- (2005). "Pedestrian tracking with shoe-mounted inertial sensors". In: IEEE Computer Graphics and Applications 25.6, pp. 38-46.

Foxlin, E., M. Harrington, and Y. Altshuler (1998). "Miniature 6-DOF inertial system for tracking HMDs". In: Proceedings of the SPIE 3362, pp. 1-15.

Friedland, B. (1969). "Treatment of bias in recursive filtering". In: IEEE Transactions on Automatic Control 14.4, pp. 359-367.

Gallagher, A., Y. Matsuoka, and Wei-Tech Ang (2004). “An efficient real-time human posture tracking algorithm using low-cost inertial and magnetic sensors". In: 2004 IEEE/RSJ International Conference on Intelligent Robots and Systems (IROS) 3.04CH37566, pp. 2967-2972.

Giansanti, D., G. Maccioni, and V. Macellari (2005). “The development and test of a device for the reconstruction of 3-D position and orientation by means 
of a kinematic sensor assembly with rate gyroscopes and accelerometers". In: IEEE Transactions on Biomedical Engineering 52.7, pp. 1271-1277.

Giansanti, D. et al. (2003). "Is it feasible to reconstruct body segment 3-D position and orientation using accelerometric data?" In: IEEE Transactions on Biomedical Engineering 50.4, pp. 476-483.

Gordon, N., B. Ristic, and S. Arulampalam (2004). Beyond the Kalman filter: Particle filters for tracking applications. Vol. 830. Artech House, London.

Hermann, T., O. Höner, and H. Ritter (2006). “AcouMotion-An Interactive Sonification System for Acoustic Motion Control". In: Gesture in Human-Computer Interaction and Simulation, pp. 312-323.

Jiménez, A. R. et al. (2010). “Indoor pedestrian navigation using an INS/EKF framework for yaw drift reduction and a foot-mounted IMU". In: 2010 7th Workshop on Positioning, Navigation and Communication, Dresden, pp. 135-143.

Kailath, T., A. H. Sayed, and B. Hassibi (2000). Linear Estimation. Upper Saddle River, NJ: Prentice-Hall.

Kay, S. (1993). Fundamentals of Statistical Signal Processing: Estimation Theory. Prentice Hall International Editions, Englewood Cliffs, NJ.

Kirk, A. G., J. F. O’Brien, and D. A. Forsyth (2005). “Skeletal parameter estimation from optical motion capture data". In: IEEE Computer Society Conference on Computer Vision and Pattern Recognition 2, pp. 782-788.

Kraft, Edgar (2003). “A quaternion-based unscented Kalman filter for orientation tracking". In: Proceedings of the Sixth International Conference of Information Fusion. Vol. 1, pp. 47-54.

Kuipers, Jack B et al. (1999). Quaternions and rotation sequences. Vol. 66. Princeton university press Princeton. 
Lapinski, Michael Tomasz (2008). “A wearable, wireless sensor system for sports medicine". In: Diss. Massachusetts Institute of Technology, School of Architecture and Planning, Program in Media Arts and Sciences.

Li, J., J. Fang, and M. Du (2012). “Error Analysis and Gyro-Bias Calibration of Analytic Coarse Alignment for Airborne POS". In: IEEE Transactions on Instrumentation and Measurement 61.11, pp. 3058-3064.

Lim, Jaechan, Myoungin Shin, and Woonjae Hwang (2017). "Variants of extended Kalman filtering approaches for Bayesian tracking". In: International Journal of Robust and Nonlinear Control 27.2, pp. 319-346.

Luinge, H. J. (2002). "Inertial Sensing of Human Motion". In: PhD Thesis, Univeristy of Twente, Enschede, The Netherlands.

Luinge, H. J. and P. H. Veltink (2004). “Inclination measurement of human movement using a 3-D accelerometer with autocalibration". In: IEEE Transactions on Neural Systems and Rehabilitation Engineering 12.1, pp. 112-121.

- (2005). "Measuring orientation of human body segments using miniature gyroscopes and accelerometers". In: Medical and biological engineering and computing 43.2, pp. 273-282.

Luinge, H. J., P. H. Veltink, and C. T. M. Baten (1999). “Estimation of orientation with gyroscopes and accelerometers". In: Proceedings of the First Joint BMES/EMBS Conference. 1999 IEEE Engineering in Medicine and Biology 21st Annual Conference and the 1999 Annual Fall Meeting of the Biomedical Engineering Society, Atlanta, GA 2, p. 844.

Lun, D. P. K. et al. (2010). “Low cost motion tracking system based on structured light illumination". In: International Symposium on Intelligent Signal Processing and Communication Systems, pp. 1-4. 
O’Brien, J. F. et al. (2000). “Automatic joint parameter estimation from magnetic motion capture data". In: In Proceedings of Graphics Interface 2000, pp. 53-60.

Rehbinder, H. and Xiaoming Hu (2001). “Drift-free attitude estimation for accelerated rigid bodies". In: Proceedings 2001 ICRA. IEEE International Conference on Robotics and Automation 4, pp. 4244-4249.

Rhudy, Matthew et al. (2009). "Microphone array analysis methods using crosscorrelations". In: ASME 2009 International Mechanical Engineering Congress and Exposition. American Society of Mechanical Engineers, pp. 281-288.

Robotics, CH (2012). AN-1008 - Sensors for Or ientation Estimation. URL: http: //www. chrobotics.com/.

Roetenberg, D. (2006). "Inertial and Magnetic Sensing of Human Motion". In: PhD thesis, University of Twente.

Roetenberg, D. et al. (2005). “Compensation of magnetic disturbances improves inertial and magnetic sensing of human body segment orientation". In: IEEE Transactions on Neural Systems and Rehabilitation Engineering 13.3, pp. 395-405.

Sabatini, A. M. (2006). “Quaternion-based extended Kalman filter for determining orientation by inertial and magnetic sensing". In: IEEE Transactions on Biomedical Engineering 53.7, pp. 1346-1356.

Sabatini, A. M. et al. (2005). "Assessment of walking features from foot inertial sensing". In: IEEE Transactions on Biomedical Engineering 52.3, pp. 486-494.

Salas, Eduardo et al. (2005). “Using Simulation-Based Training to Improve Patient Safety: What Does It Take?" In: The Joint Commission Journal on Quality and Patient Safety 31, pp. 363-371. URL: http:// dx . doi . org/10 . 1016 / S1553-7250 (05) 31049-X. 
Simon, Dan (2006). Optimal state estimation: Kalman, H infinity, and nonlinear approaches. John Wiley \& Sons.

Wan, Eric A and Rudolph Van Der Merwe (2000). "The unscented Kalman filter for nonlinear estimation". In: Adaptive Systems for Signal Processing, Communications, and Control Symposium 2000. AS-SPCC. The IEEE 2000. Ieee, pp. 153158.

Yun, X. and E. R. Bachmann (2006). “Design, Implementation, and Experimental Results of a Quaternion-Based Kalman Filter for Human Body Motion Tracking". In: IEEE Transactions on Robotics 22.6, pp. 1216-1227.

Yun, X., E. R. Bachmann, and R. B. McGhee (2008). “A Simplified QuaternionBased Algorithm for Orientation Estimation From Earth Gravity and Magnetic Field Measurements". In: IEEE Transactions on Instrumentation and Measurement 57.3, pp. 638-650.

Zhang, L. et al. (2011). “Building Virtual Entertainment Environment with Tiled Display Wall and Motion Tracking". In: International Conference on Virtual Reality and Visualization, Beijing, pp. 283-286.

Zhou, H., H. Hu, and Y. Tao (2006). “Inertial measurements of upper limb motion". In: Medical and Biological Engineering and Computing 44.6, pp. 479-487. 